\title{
Microstructure metrics for quantitative assessment of particle size and dispersion:
}

\section{Application to metal-matrix composites}

\author{
Mohammad Jahedi $^{\text {a,* }}$, Ehsan Ardjmand ${ }^{\text {b }}$, Marko Knezevic ${ }^{\text {a }}$ \\ ${ }^{a}$ Department of Mechanical Engineering, University of New Hampshire, Durham, NH 03824, \\ USA. \\ ${ }^{\mathrm{b}}$ College of Business, Department of Management, Frostburg State University, Frostburg, MD \\ 21532, USA.
}

\begin{abstract}
Homogeneous dispersion of reinforcement particles within a matrix is of paramount importance for achieving high quality metal-matrix composite (MMC) materials. This paper develops a protocol for quantitative evaluation of particle size and dispersion in $\mathrm{MMC}$ microstructures. The protocol is based on statistical analysis of features in micrographs and embedding the information into a suitably defined microstructure homogeneity metric ( $H$-metric) and a particle size distribution metric. Every micrograph undergoes the $K$-means clustering algorithm for accurate separation of phases before it is used in calculation of the metrics. Finally, the protocol relies on analysis of variance to verify the meaningfulness of results. The protocol is applied to study homogeneity and particle size distribution in MMCs consisting of Cu-matrix with 20vol.\%-SiC The composites were processed using high pressure torsion and double torsion to a different number of torsional turns. During processing, particles fragment and form clusters of fragments. As straining continues, the clusters of fragments disperse in the matrix. A decrease and then increase of the $H$-metric demonstrates that the metric can pick up these processes occurring in the microstructure. The analysis reveals a strong correlation between the particle dispersion homogeneity and particle size distribution. The protocol developed herein can facilitate the design of MMCs with superior properties through engineering optimal homogeneity and particle size distributions in function of process variables.
\end{abstract}

\footnotetext{
${ }^{*}$ Corresponding author at: Department of Mechanical Engineering, University of New Hampshire, 33 Academic Way, Kingsbury Hall, W322, Durham, NH 03824, USA.

E-mail address: mohammad.jahedi@unh.edu (M. Jahedi).
} 
Keywords: Metal-matrix composites (MMCs); Microstructural homogeneity; Severe plastic deformation (SPD); $K$-means algorithm; Analysis of variance (ANOVA)

\section{Introduction}

A mathematical framework termed microstructure sensitive design (MSD) has been conceived to quantitatively describe microstructure, evolve microstructure during processing, and treat it as a continuous design variable in engineering design and optimization. MSD can predict mechanical properties while featuring invertible linkages between the statistical description of a microstructure and its effective properties [1,2]. The framework was largely focused on the statistical description of crystal orientations using orientation distribution functions (ODFs) as the primary metric for microstructures [1-5]. This paper presents an extension of the previously developed microstructure metrics for quantitative description of microstructures in the statistical sense to enable assessment of particle size and dispersion in metal-matrix composites (MMCs).

MMCs are a class of material systems consisting of at least two constituent materials, one of which is a metal. Homogenous dispersion of reinforcement particles and their size within a matrix are key features determining the quality of these materials. Therefore, quantitative microstructure metrics and protocols for their evaluation in function of process parameters are critically needed for cost-effective and fast design of improved or new MMCs. New materials have a great potential for impacting virtually all emerging technologies, with significant economic consequences. The central impediment in the computational design of materials and processes comes from the need to consider the relevant details of the hierarchical internal structure that controls the properties of interest to a specific application [6-9]. The present work aims at developing a protocol to judiciously identify the effect of processing parameters on the homogeneity of the reinforcements in MMCs to facilitate quantitative comparisons of microstructures, and eventually to reduce the underlying cost and number of experiments currently required to synthesize MMCs of superior properties.

MMCs can be synthesized via powder metallurgy route [10-13]. Depending on the particle size of the powders and the mixing method, this process often produces composites with a low uniformity of the reinforcement material as well as weak adhesion of the reinforcement 
and the metal matrix $[14,15]$. Thermomechanical (introducing heat during the plastic deformation) steps are usually applied to the mixture to improve the dispersion of the reinforcement material within a metal matrix. This process continuously mashes the components together and breaks the mixture apart over and over again to disperse powder particles [16, 17]. Mixture homogeneity is essential for obtaining MMCs with a high degree of homogeneity. The mixing processes ideally prepare a "random mixture," where all components are uniformly distributed in the mass. This is difficult to achieve in practice, especially when dealing with components of widely different properties as in the MMCs presented here [18, 19]. The most common definition of a perfectly random mixture is one in which the probability of finding a particle of a constituent of the mixture is the same for all points in the mixture [20].

The clustering of the particulate reinforcement during MMC production has an important influence on MMC properties. This is undesirable as it leads to non-homogeneous response, leading to strain localizations and ultimately premature failure [21]. In cases where the particles are well dispersed, the interaction of particles with each other at higher volume fractions can cause reduced particle settling rates leading to clustering. Particle clustering can occur due to chemical bonding, reduction of surface energy, or segregation of the particles especially when the size of the particles is small. In addition to particle dispersion, target properties of MMCs are achieved by influencing their particle size distribution. To this end, a combination of finer particulate size distribution and high homogeneity reduces mean free path of mobile dislocations between particles within a ductile matrix, which results in MMCs of high strength and stiffness. Achieving the finer particle size in composites enables the reduction of the cost involved in manufacturing the MMCs' parts by downstream steps such as extrusion and machining, as finer particles introduce better formability in the composites [22]. Small particles in MMCs can improve both ductility and strength in comparison to those that contain larger particles [23, 24]. In contrast, fine particles can reduce surface properties such as wear resistance [25, 26].

To achieve random dispersion of particles within MMCs, severe plastic deformation (SPD) processes have attracted much attention due to their ability to improve the homogeneity of particle distribution with plastic strain [15, 27]. Conventionally, rolling and extrusion processes have been used extensively [24, 27]. It was found that improving the homogeneity requires very high strains $(>4)$, especially for composites containing fine particles. The efficiency at which 
these techniques can improve the homogeneity depends on the level of strain possible to impose on a material system by a technique. While SPD processes such as equal channel angular pressing (ECAP) [28, 29] and accumulative roll bonding (ARB) [30-32] have been found effective for improving the distributions, the best particle distribution thus far was obtained using high pressure torsion (HPT) processing [33]. Recently, an extension of the HPT process called high pressure double torsion (HPDT) was introduced [11, 34-36] as a way of increasing the amount of strain imposed per turn, and hence the grain size refinement was more efficient compared to standard HPT [34, 36, 37]. In this study, HPT and HPDT are used to manufacture the composite materials. In these processes, there is a strain gradient inside a sample, and the strain grows as a function of radius. Number of turns is one of the most influential parameters in these processes as more strain is applied with number of turns.

MMCs containing reinforcement particles are so-called segregated mixtures, where the particle phase segregates by differences in the size and properties such as density and strength. Homogeneity of microstructure in MMCs can be assessed based on image analysis. To this end, several procedures have been developed in the literature. In these procedures, it is convenient to define macro-pixels as non-overlapping entities of appropriate size dividing an image into $N$ usually square entities. The procedures then compare statistics of each macro-pixel with the statistics of whole images [23, 38, 39]. In these works, the theoretical variance of a binary mixture in the segregated state (non-homogeneous) is conveniently expressed by

$$
\sigma_{0}^{2}=p(1-p)
$$

and the random or homogeneous state is formulated by

$$
\sigma_{r}^{2}=\frac{p(1-p)}{n}
$$

where $p$ and $n$ are the weight percentage and number of reinforcements, respectively [20].

A segregation intensity $(S)$ was introduced by Dankwertz [40] in 1952, as a measure of uniformity in solid mixtures. The original index and its image-adapted version are defined by

$$
S=\frac{\sigma^{2}-\sigma_{r}^{2}}{\sigma_{0}^{2}-\sigma_{r}^{2}}
$$


In Eq. (3), $\sigma$ is a standard deviation of a grid on the micrographs. $S$ value of 1 corresponds to a segregated state while 0 to a totally random state.

As an alternative, Lacey introduced the mixing index (M) to assess mixture homogeneity [41]. The index or its image-adapted version is defined by [20],

$$
M=\frac{\sigma_{0}^{2}-\sigma^{2}}{\sigma_{0}^{2}-\sigma_{r}^{2}}
$$

Like $S$, M ranges from 0 (segregated) to 1 (random). Despite being useful for low content particles in a matrix, it is shown that these indexes do not always perform well for binary images that have a high content of second phase [42].

Several other methods such as scenario analysis [43], structural equation [44], neural networks [45, 46], and Monte Carlo simulations [47] can also be useful in quantifying the homogeneity. However, these methods are not suitable for the present study's purpose for several reasons. Scenario analysis is usually used when various possible future events are to be analyzed, while in this study only one scenario under different incorporated process variables' values is considered. Structural equation method is more appropriate for cases where there are many input parameters that have interaction and causality relation between these parameters is not totally known [48]. Artificial neural networks (ANNs), while being very useful for modeling complicated systems, are considered a black-box method due to their lack of ability in explaining the relationship between parameters of a system [49, 50]. Also, training ANNs is computationally expensive, and it may be necessary to use complicated algorithms to obtain desirable results $[50,51]$. Hence, ANNs cannot be used for this study where the focus is on understanding the relationship between the systems' variables and homogeneity. In Monte Carlo simulation [52], knowledge of the distribution of the variables is needed, which is not the case in this study. Analysis of variance (ANOVA) can be used to examine input data sets due to its ability to explain the interaction between systems parameters and the target incorporated process variables. The ANOVA method has been used for the design of experiments in manufacturing where the effect of manufacturing variables of interest on a target variable was evaluated [53, 54]. The target variable was preselected based on its significant influence on the given process. 
In this work, homogeneity of reinforcement dispersion within a sample, i.e., along the sample radius, and as a function of number of torsional turns was studied in samples synthesized using HPDT and HPT. In particular, we aimed at determining the effect of number of turns as a manufacturing parameter on the microstructural homogeneity and particle size distribution. $K$ means algorithm was utilized to convert micrographs into the most representative binary single image with constituent phases accurately identified. Next, homogeneity and particle size distribution at a given radius per sample were quantified using a suitable defined microstructure homogeneity metric termed the $H$-metric and a particle size distribution metric. Finally, ANOVA method was used to examine the validity of the results. It is demonstrated quantitatively that significant improvements in the homogeneity of the hard particle dispersion can be obtained using HPT and HPDT. It is determined that reinforcements are fragmenting and the fine particles are dispersing faster in the HPDT process than in HPT. This is attributed to the large strains and strain rate imposed by the HPDT process. However, we find that the homogeneity can reduce as the particles are constantly breaking (reducing in size), dispersing in matrix from broken clusters, and subsequently clustering again in matrix at very high strain levels achieved by HPDT. We further observe a strong correlation between the particle dispersion homogeneity and particle size distribution. It is concluded that homogeneity ( $H$-metric) and size distribution must be simultaneously assessed when determining the quality of microstructure in MMCs. The protocol developed herein can be applied to search an optimal homogeneity and particle size distribution in MMCs as a function of process variables.

\section{Materials and experimental approach}

Commercially pure Copper $(\mathrm{Cu})$ and Silicon-Carbide $(\beta-\mathrm{SiC})$ particulate powders with average particle sizes of 30 and $5 \mu \mathrm{m}$, respectively were used as starting materials. Both $\mathrm{Cu}$ and SiC powders were supplied by Sigma-Aldrich, Germany. The powders were of high purity (99.9\%). The mixing of the $\mathrm{Cu}$ and the 20 vol.\% of SiC powders was carried out by jar milling for $30 \mathrm{~min}$. The mixed powders were consolidated under HPT and HPDT with pressure of 1.8 $\mathrm{GPa}$ and an anvil rotation rate $(\omega)$ of $0.2 \mathrm{rpm}$ at room temperature under 2,4 , and 6 turns. In the HPDT technique, the rotation rates for the top and bottom anvils were equal. More details of the experimental procedure can be found in [11]. 
To investigate the particle dispersion in the composite samples, micrographs were taken using scanning electron microscopy (SEM). The characterization was performed in an Amray 3300FE field emission SEM operated at $7 \mathrm{kV}$ in secondary electron (SE) mode imaging. For each instance, i.e., center, middle, and edge, 10 micrographs were taken, resulting in 30 micrographs for each composite sample. In Fig. 1, one out of ten of initial micrographs are shown at middle and edge locations of HPDT and HPT samples at 2 and 6 number of turns. By visual inspection, it is not possible to determine the level of homogeneity in the samples. A quantitative analysis is necessary to identify the most homogeneous microstructure.

During severe plastic deformation imparted by HPT and HPDT on the MMC, damage accumulates usually at the interface between $\mathrm{SiC}$ particles and the matrix because of large strain incompatibilities at these locations. In addition to de-cohesion at the particle/matrix interface, damages can initiate by reinforcement breaking. Particle breaking occurs less in composites containing fine particles $[55,56]$. Figure 2 illustrates several instances of void initiation. Poor dispersion of SiC particles within the matrix leads to rapid failure of the composite. Upon void formation, void propagation through the matrix is usually catastrophic.

The $\mathrm{Cu}$ matrix is ductile and able to accommodate large imposed strains. In contrast, the hard SiC particles are brittle, and fracture under imposed deformation $[55,56]$. The overall composite can withstand larger strains to failure, since the breakage of particles occurs locally and gradually. In HPT and HPDT to high number of turns, the strain levels are extremely high in magnitudes of 10 and significantly more. The material typically exhaust hardening very early in the process. At this point, the matrix continues to accommodate the imposed plastic strain with very little to no hardening. Due to the difficulties with estimating magnitudes of stress and strain, we based our results on the process variable (number of turns). The hydrostatic pressure of matrix on the particles prevents creation of cracks and at the same time helping the existing cracks not to extend within the matrix. The shear due to plastic deformation breaks agglomerations as well as particles. Moreover, during HPT or HPDT, the created voids are collapsing. Local tensile stresses develop and act on particulates. These stresses are a consequence of local interactions, where the load is transferred from matrix to particles. Larger particles fracture first because the probability of finding defects in particles increases with the particulate size. Among the particulates of approximately the same size, those with smaller 
aspect ratio (more elongated) and oriented with their major axis along the loading direction are subjected to higher stresses and more likely to fail earlier during deformation because the load transfer from the matrix to them is more efficient. Examples of particle fragmentation during HPDT and HPT are depicted in Fig. 3. Arrows indicate the cracks.

After fragmentation, the fragments constitute a cluster of particles of smaller size than the parent particle. Experimental findings show that there is a relationship between damage formation and the local volume fraction of the clusters. Here, de-bonding and crack initiation occurs around the perimeter of clusters. Subsequently, the plastic flow of the matrix inside the cluster initiates during the plastic flow of the matrix in the regions without clusters. This plastic flow of ductile matrix fills up the space between the particles inside a cluster with the matrix material. During continuous straining, the clusters de-agglomerate within the matrix as the matrix plastically deforms. Thus, the homogeneity of the material improves. In Fig. 4, the instances of void formations and cracks after fragmentation of a particle are shown in both HPT and HPDT processes. It should be noted that particle size distribution is continuously shifting towards the smaller mean value and larger standard deviation as the straining continues. In the case of poor dispersion, the finer reinforcements could weaken the composites more than the coarser reinforcement $[57,58]$. As the reinforcement's size decreases, the matrix grain size also decreases [59, 60]. In the case of good dispersion, the finer particle size results in a greater strength and hardness of the composite [61]. The decrease in the reinforcing particles' size was found to improve dynamic Young's modulus and damping capacity [62] as well as low cycle fatigue behavior of discontinuously reinforced MMCs [63]. Despite these facts, a larger particle size distribution was found to enhance surface properties better than the smaller one in those applications requiring properties such as wear resistance $[25,26]$. In particular, abrasive wear is improved with larger particles because smaller particles break free much easier than bigger ones. The free particles are debris that slide between surfaces and remove the material, which is undesirable. Tools for quantitative understanding of effects of processing on microstructure in MMCs can guide the design of microstructures in these materials for target performances. 


\section{Protocol and metrics}

This section presents the framework developed for quantitative description of phenomena described in the previous section facilitating quality assessments and comparisons of microstructures in MMCs processed under different process conditions.

\subsection{K-means clustering algorithm}

The first step to analyze the homogeneity of $\mathrm{SiC}$ particles is to identify them in the images. For this purpose, it is necessary to convert the greyscale images obtained into binary images where the $\mathrm{SiC}$ and $\mathrm{Cu}$ particles are represented with the values of 0 and 1 (black and white) respectively. A pixel in a greyscale image only contains the intensity information and can have any value between 0 to 255 . The weakest and strongest intensity values belong to black and white colors respectively with black having the value of 0 and white 255 . Figure 5 shows a greyscale image and its pixels' values distribution.

As can be observed in Fig. 5, the pixels of a greyscale image can have any value between 0 to 255 and hence, to detect the $\mathrm{SiC}$ particles, it is important to determine a threshold between particles based on their representative greyscale pixel values. To cluster the particles into $\mathrm{SiC}$ and $\mathrm{Cu}$, a $K$-means clustering algorithm [64-66] is used. $K$-means is a widely used algorithm for clustering data and has many applications in data mining [67]. We utilize the $K$-means algorithm implemented in the $\mathrm{R}$ data analysis software [68].

The inputs to the $K$-means algorithm are a set of $\mathrm{n}$ data points (in d dimensional space) and an integer $K$ which specifies the number of clusters in data. The objective of $K$-means is to minimize the mean squared distance between each data point to the center of each cluster. If a pixel in the greyscale image is represented by $x_{m n}$, where $m$ and $n$ are the coordinates of the pixel, the $K$-means algorithm minimizes

$$
Q=\sum_{k=1}^{K} \sum_{m n=1}^{M N}\left\|x_{m n}^{k}-m_{k}\right\|^{2}
$$

where $x_{m n}^{k}$ is the pixel $m n$ assigned to cluster $k$ and $m_{k}$ is the center of the cluster $k$. Having fixed the value of $K$, the $K$-means algorithm determines $m_{k}$ values and changes them in each step while minimizing $Q$. 
Using this method, it is possible to segment the pixels based on their greyscale values into two separated clusters representing $\mathrm{SiC}$ and $\mathrm{Cu}$ particles. Note that in this study $K$ is set to 2 to cluster the image identifying the two phases, namely $\mathrm{SiC}$ and $\mathrm{Cu}$. After clustering the pixels, a binary image can be produced based on the greyscale image that can be further used in the homogeneity analysis. It should be noted that the cracks and voids were detected based on their different greyscale colors and these were then converted to the matrix color.

The results of applying $K$-means to the binary images show that on an average $22 \%$ of the pixels in an image represent the SiC phase. The original mixture contained $20 \%$ of SiC. The fact that the two percentages are close to each other indicates that the number of SEM images (10 images per case) was enough to be statistically significant. The reason for this difference is the projection of a 3-dimensinal object (the mixture in this case) into a 2-dimensional image. To explain this, it is helpful to consider a sphere with radius $r$ that is circumscribed in a cube with side $2 r$. The ratio of sphere volume to the cube would be $\frac{\frac{4}{3} \pi r^{3}}{8 r^{3}}=\frac{\pi}{6}$. The objects obtained by projecting the sphere and the cube to a 2-dimensional space would be a circle with radius $r$ and a square with side $r$. Now, the ratio of the circle area to the square area would be $\frac{\pi r^{2}}{4 r^{2}}=\frac{\pi}{4}$ which less than the volume ratio is. This example shows that having a volume ratio does not necessarily imply having the same ratio when looking at a 2-dimensional projection of volume. Hence, it is possible to observe a higher ratio of $\mathrm{Cu}$ to $\mathrm{SiC}$ in a binary image compared to the original volume mixture.

\subsection{Homogeneity metric (the H-metric)}

The homogeneity measurement method used in this research is based on the approach introduced by Poole et al. [69] and applied to image processing by Rosas and Blanco [42]. The basis of the homogeneity measurement method used in this research compares the sample binary image with a specialty built one where the pixels representing $\mathrm{SiC}$ and $\mathrm{Cu}$ particles are distributed randomly. A random image is representative of an ideal mixture of $\mathrm{Cu}$ and $\mathrm{SiC}$, where each pixel is randomly assigned a value of 1 or $0(\mathrm{SiC}$ or $\mathrm{Cu})$. The random image is used to set a baseline for comparing the images. $22 \%$ of $\mathrm{SiC}$ is ensured to be present in this random image. Note that the homogeneity is calculated for each sample using 10 binary micrographs at three locations. For this purpose, the binary images (of the HPT and HPDT sample as well as the 
specialty built one as random) are divided into $N=121$ non-overlapping macro-pixels after selecting the edge of each macro pixel to be $20 \mu \mathrm{m}$. This size is suggested in $[11,39,70]$ as the optimal size, corresponding to 4 times the size of the mean particle size. In HPT and HPDT processes, the reinforcements are continuously breaking into smaller particles with the applied plastic deformation. This may suggest that for every strain level, a new macro-pixel size (a smaller one) should be considered. A few examples illustrating the different macro-pixel sizes are presented in Fig. 6. A cluster introduced by fragmentation of a particle is always larger than the original particle. Specifically, the figure illustrates two clusters (shown by arrows). With the macro-pixel size shrinking, the particles of a cluster are filling multiple macro-pixels instead of belonging to a single coarse macro-pixel. Thus, this particular cluster may not be identified using a small macro-pixel. In addition, we have performed the calculations by varying the macro-pixel edge from 10 to $40 \mu \mathrm{m}$ in increments of $2 \mu \mathrm{m}$. While the results showed fluctuations in the $H$ metric values, the overall trends in the $H$-metric were identical.

Figure 7 shows the procedure for calculating the $H$-metric. Each macro-pixel on the binary image as well as on the full image is a matrix of 0 and 1 values. For every macro-pixel, the total number of pixels that have value equal to 1 (white) is divided by the total number of pixels (this ratio is the mean value for the given macro-pixel). In Fig. 8, examples of the macropixels for a real micrograph and a random binary image as well as the associated distributions and mean and standard deviation (Sd) values of the distributions are presented. For comparison, the distributions of whole images are also provided. The whole random image and the macropixels from the random image have the mean and standard deviation values very close to each other. In contrast, the macro-pixels extracted from the micrograph exhibit differences in terms of the statistical measurements.

Each macro pixel can be considered as a standalone sample from a larger population (the whole image), thus the distribution of phases in macro-pixels can be compared with those in the whole image. For each macro-pixel, there is a Sd value, in addition to a mean value. A value of $\mathrm{Sd}$ close to 0 indicates a high particle depletion. The opposite ( $\mathrm{Sd}$ close to 1 ) indicates a high particle segregation. A mean value of the standard deviations over the macro-pixels belonging to a micrograph as well as belonging to the random image can be calculated after the Sd values of 
individual macro-pixels are known. Then, the homogeneity metric for a sample at a given location can be calculated as:

$$
H=\frac{\bar{G}_{S d}}{\bar{G}_{\text {sd_random }}},
$$

where $\bar{G}_{s d}=\frac{\sum_{i=1}^{N} s d_{i}}{N}$ is average standard deviation of individual macro-pixel distributions, and $\bar{G}_{s d_{-} \text {random }}=\frac{\sum_{i=1}^{N} s d_{i_{\text {rrandom }}}}{N}$ is average of standard deviation of macro-pixel distributions from the random image, where $N=121$. This procedure was repeated for all ten images at a given location per sample and the average of these ten $H$-metric values were then reported as the $H$ metric value for that location. Note that the $H$-metric captures the homogeneity without explicitly considering the size of particles. If in a region of the image the particles are larger, $\bar{G}_{s d}$ of that region will implicitly reflect this fact and the $H$-metric will change accordingly.

\subsection{Analysis of variance (ANOVA) test}

To evaluate the effect of number of turns and radius on the homogeneity, (ANOVA) is used. ANOVA is available in commonly used statistical analysis tools such as MATLAB and Microsoft Excel. ANOVA is a statistical method for analyzing the differences among samples in terms of average and variability, which is the extent to which data points in a statistical distribution or data set diverge from the average value as well as the extent to which these data points differ from each other. The average and the variability are expected to be different for every studied sample in the present study. Every sample is processed under a different value of the number of turns and the microstructure varies with radius. The ANOVA test starts by assuming there is no significant difference between the sample averages (null hypothesis) while the input values - i.e., number of turns and radius - are changing. Then, assuming the null hypothesis holds, the probability of observing the obtained results is evaluated. If this probability $(P$-value) is lower than a predefined threshold, it shows that the chance alone cannot produce such a result and hence, the null hypothesis might not statistically hold. In this case, it makes sense to conclude that the averages are different and this difference is due to the changes in input values. 
To decide whether to reject or accept a hypothesis using ANOVA, one needs to compare the $P$-value of the test with the significance level, which is a predefined value usually set to 0.05 [71]. Note that the $P$-value is the probability of obtaining a test statistic that is at least as extreme as the actual calculated value, if the null hypothesis is true. For example, if the $P$-value equals to 0.8, it means that assuming all statistical assumptions hold (i.e., null hypothesis is correct) and the test is conducted many times, the chance of observing the obtained results (in this study, homogeneity, $H$-metric value) is $80 \%$.

It should be noted that the amount of $P$-value can also show the effect of a variable on a response. A lower amount of $P$-value is attributed to higher importance of that variable [72]. $P$ value amounts lower than a prescribed value indicate that the studied parameter is significant in the results of the phenomenon [73]. The interested reader is referred to [71, 74, 75] for more explanation regarding the ANOVA testing.

\subsection{Particle size distribution metric}

Particle size is measured using an extended intercept length method. Here the standard intercept length method is extended for the characterization of particle size variation by including the particles' boundaries. For an improved region representation, the isolated regions (the particles do not share boundaries with the others) are filled with a value different from the nearest neighboring point. A MATLAB code tracks the boundaries and identifies the neighboring boundaries and considers measurements smaller than three pixels as noise and thus excludes them. After capturing the isolated particles, the code estimates the area of all the pixels in an isolated region by summing the areas of each pixel in that region. The area of an individual pixel is determined by looking at its 2-by-2 neighborhood. The equivalent size of a particle is the diameter of a circle with the same area as the measured isolated region.

\section{Results and discussion}

\subsection{H-metric}

In Table 1 , the average $H$-metric values for the HPDT and HPT processes were tabulated as a function of number of turns as well as the variations of this metric by radius, i.e., at center, middle, and edge locations on the specimens. The corresponding $P$-values for the named 
comparisons were also tabulated. The null hypothesis decisions based on the ANOVA tests are also included as "Accepted" and "Rejected" terms to study the statistical significance of number of turn and radius on varying $H$-metric values. The comparisons are as following: (1) "\# of turns varies at constant radius" means that the $H$-metric values of the number of turns 2,4 , and 6 are compared together at the specified locations mentioned by Center, Middle, and Edge; and (2) "Radius varies at constant \# of turns" means that $H$-metric values along the radius (from center to edge) are compared at specific number of turns 2,4 , and 6.

The $P$-values for the HPT process in all cases are over 0.05. This explains that there is no evidence that the number of turns and radius can influence the $H$-metric in the HPT process. In other words, regardless of the amount of strain imposed, statistically there is no proof to conclude that increasing the strain influences the homogeneity in the HPT samples. The same comparison for HPDT shows that in all cases the $H$-metric values are less than 0.05 except the one that compares the effect of number of turns at the center location. It can be concluded with confidence that HPDT can evolve the $H$-metric in a statistically significant manner. In Fig. 9, the average $H$-metric values of the HPDT process were plotted. In Fig. 9a, the $H$-metric values at 2 turns show that the homogeneity decreases from edge to the center, which can be expected due to the intrinsic decrease in the strain gradient inside the sample from edge to the center. After 4 turns, the $H$-metric values at all locations are decreasing, while with the continuation of deformation to 6 turns, the $H$-metric values are increasing.

At the center location, the fluctuation in $H$-metric values is the least since the imposed strain at this location is the smallest, while in other locations the displacement of the particles is substantially more pronounced due to higher strain levels, causing the $H$-metric values to significantly change.

In Fig. 9b, after 2 turns there is an increasing trend in $H$-metric values from the center to edge. After 4 turns, the $H$-metric values drop at all locations. Subsequent straining from 4 to 6 turns results in an increase in homogeneity. Examining the effect of turn shows that increasing the number of turns reduces gradients in one sample amongst the homogeneity at different locations of that sample. However, the $H$-metric values at 6 turns is less than that at 2 turns. Thus, there is a competition between the $H$-metric values at every location and the gradient of $H$ metric values in a sample. 


\subsection{Particle size distribution}

In HPT and HPDT, there is an intrinsic strain gradient along the sample radius. Thus, gradients in particle size and homogeneity are expected in every sample along the radius. Moreover, more turns impose more strain on the sample, creating a dependency of particle size distribution on number of turns. In Fig. 10, the size distribution histograms are presented for samples created using HPT and HPDT to different turn numbers at the three locations per sample. After 2 turns of HPT, the size distribution histograms show a smaller particle size at the center than at the middle and edge locations which can be justified by the presence of clusters in the microstructure. The particles displacements enhance by a higher strain value (higher strain value at middle and edge locations). This causes the particle to be initially agglomerated as it is assumed that at this strain level, the strain is not large enough to break the particles to finer particles. Hence, the center location which received less strain shows finer particle size (less agglomerated areas). As the deformation continues (Fig. 10b), the amount of strain is high enough to break the agglomerated particles, moving them apart from each other. The edge location shows the smallest size distribution in comparison to the center and middle locations. This finding corresponds with the $H$-metric values in Table 1 . The $H$-metric values for 4-turn are higher than those for 2 turns. After 6-turn HPT (Fig. 10c), the 3 locations' size distributions received sufficient strain to reduce the size distribution gradient in the microstructure. However, as the particle size is refined, the $H$-metric values drop, which suggests that dispersing finer particles is more difficult than dispersing larger particles. In Fig. 10d, the size distribution in the material processed using the HPDT process is shown after 2 turns. The distributions appear to be similar, meaning that clusters are not present as much as in the material processed using 2-turn HPT. It is worth mentioning that in HPDT the strain value is theoretically twice as much as that in HPT. This directly translates to displacing particles at least two times faster in HPDT than in HPT. This enhances the particles' dispersion and breaks the clusters faster. After 4-turn HPDT (Fig. 10e), the amount of strain was large enough that the clusters were broken. The fact that the 2 microns' bin was filled demonstrates that the particles are significantly fragmented. The $H$ metric values dropped (Fig. 9a).

Continuing the plastic deformation in the HPDT process to 6 turns (Fig. 10f), the density of the smallest bin size ( 2 microns) drops relative to those calculated at 4 turn and the densities in 
other bins become higher. This suggests that the small size particles are creating clusters. Rebonding is unlikely because $\mathrm{SiC}$ particles do not create diffusional bond at room temperature even under very high pressures. Despite this fact, examining the trend of H-metric values at 6 turns shows that at all locations the homogeneity increases. To justify this observation, the 6-turn strain level is apparently enough to fragment large particles and to displace fragments but not enough to move apart the small particle clusters. Additionally, small particles could form new clusters during large deformation influencing the size distribution. In general, the former phenomenon is increasing the $H$-metric but the latter is decreasing it. The competition between these two phenomena results in increasing the $H$-metric at 6-turn, which means that displacement of larger particles has more effect on the H-metric than displacing smaller particles.

The corresponding cumulative distribution functions (CDFs) are presented in Fig. 11 to facilitate easy comparison of homogeneity in the size distribution. The size distributions at different locations in the material processed using two HPT turns are close to each other. The HPT 4-turn material exhibits a dramatic particle fragmentation shift at the edge location, meaning that 4 turns HPT are sufficient to start the particle fragmentations but not to displace clusters since the minimum size obtained did not change appreciably from that observed in the 2turn HPT material. At 6-turn (Fig. 11c), the three locations' distributions reached close to each other, and from the size distribution point of view there is a high level of homogeneity. In HPDT 2-turn (Fig. 11d), the homogeneity in the specimen's locations are higher than HPT 2-turn, which means more strain induces more size distribution homogeneity. As the deformation continues (Fig. 11e), difference between size distribution at the locations appears. 4 turns were sufficient to start a significant particle fragmentation. In HPT 4-turn larger particles broke down but in HPDT even smaller particles broke down. At 6-turn HPDT, the distribution is more homogenized, and the difference between different locations has reduced. The degree of homogeneity regarding the size distribution in HPT and HPDT 6-turn is similar, though in HPDT 6-turn homogeneity is achieved for a smaller particle size.

Considering that HPT 4-turn and HPDT 2-turn are theoretically identical, a comparison between their $H$-metrics and size distributions at center, middle, and edge locations is presented in Fig. 12. Examining the size distribution of these two examples suggests that these two conditions are achieving similar trends on the composite samples. Specifically, these two 
processes have some differences at the middle location where HPDT 2-turn creates more particle fragmentation than HPT 4-turn. Looking at the $H$-metrics shows that HPDT 2-turn achieves a higher homogeneity value than HPT 4-turn. To investigate the significance of this phenomenon statistically, ANOVA tests were performed at center, middle, and edge locations. The resultant $P$-values of these tests were tabulated in Table 2 . We conclude that with the current micrographs and corresponding statistical populations, the $H$-metric is not sensitive enough to capture the difference of these conditions, although the $H$-metric values in HPDT promise a higher homogeneity than HPT. To influence $P$-values, either the $H$-metric must be redefined or processing methods of HPT/HPDT must be continued to a higher strain level.

\section{Conclusion}

In this study, a protocol involving two microstructure metrics for measuring the homogeneity of the dispersion of particles in composite materials is developed. Additionally, the protocol relies on the $K$-means algorithm to preprocess the SEM micrographs and the analysis of variance (ANOVA) method to confirm the meaningfulness of the results. An interesting correlation is demonstrated between the reinforcement's dispersion homogeneity and particle size distribution in microstructures produced using two severe plastic deformation processes (SPD) of HPT and HPDT. The strain imposed by SPD processes improves the $H$-metric as long as the shearing causes the particles to fragment and displace in the matrix. Continuous straining can lead to undesirable clustering of fine particles and decreases in the $H$-metric. The protocol can facilitate achieving a compromise between particle dispersion and fine particle size in MMCs. In the HPDT process, 4 turns develop a microstructure to be optimal in this regard. The protocol shows that more strain after the 4 turns will deteriorate the $H$-metric value, although these higher strain levels could warrant a finer particle size distribution. The protocol developed in this work warrants further development to enable predicting homogeneity in yet unprocessed microstructures based on the known homogeneity of processed microstructures as a function of incorporated process variables for HPT and HPDT processes.

\section{Acknowledgement}

This work is based upon a project supported by the U.S. National Science Foundation under grant No. CMMI-1541918. The authors gratefully acknowledge this support. 


\section{References}

[1] B.L. Adams, A. Henrie, B. Henrie, M. Lyon, S.R. Kalidindi, H. Garmestani, Microstructuresensitive design of a compliant beam, Journal of the Mechanics and Physics of Solids, 49 (2001) 1639-1663.

[2] S.R. Kalidindi, J.R. Houskamp, M. Lyons, B.L. Adams, Microstructure sensitive design of an orthotropic plate subjected to tensile load, International Journal of Plasticity, 20 (2004) 15611575 .

[3] X. Wu, G. Proust, M. Knezevic, S.R. Kalidindi, Elastic-plastic property closures for hexagonal close-packed polycrystalline metals using first-order bounding theories, Acta Materialia, 55 (2007) 2729-2737.

[4] M. Knezevic, N.W. Landry, Procedures for reducing large datasets of crystal orientations using generalized spherical harmonics, Mechanics of Materials, 88 (2015) 73-86.

[5] S.R. Kalidindi, J.R. Houskamp, Application of the Spectral Methods of Microstructure Design to Continuous Fiber Reinforced Composites, Journal of Composite Materials, 41 (2007) 909-930.

[6] D.B. Brough, D. Wheeler, J.A. Warren, S.R. Kalidindi, Microstructure-based knowledge systems for capturing process-structure evolution linkages, Current Opinion in Solid State and Materials Science, (2016).

[7] S.R. Kalidindi, Data science and cyberinfrastructure: critical enablers for accelerated development of hierarchical materials, International Materials Reviews, 60 (2015) 150-168.

[8] G.B. Olson, Designing a new material world, Science, 288 (2000) 993-998.

[9] J. Allison, Integrated computational materials engineering: A perspective on progress and future steps, Jom, 63 (2011) 15.

[10] H. Zare, M. Jahedi, M.R. Toroghinejad, M. Meratian, M. Knezevic, Compressive, shear, and fracture behavior of CNT reinforced Al matrix composites manufactured by severe plastic deformation, Materials \& Design, 106 (2016) 112-119.

[11] M. Jahedi, M.H. Paydar, M. Knezevic, Enhanced microstructural homogeneity in metalmatrix composites developed under high-pressure-double-torsion, Materials Characterization, 104 (2015) 92-100.

[12] A. Canakci, T. Varol, Microstructure and properties of AA7075/Al-SiC composites fabricated using powder metallurgy and hot pressing, Powder Technology, 268 (2014) 72-79.

[13] M.R.P. Fernandes, A.E. Martinelli, A.N. Klein, G. Hammes, C. Binder, R.M. Nascimento, Production of nickel matrix composites reinforced with carbide particles by granulation of fine powders and mechanical pressing, Powder Technology, 305 (2017) 673-678.

[14] S. Amirkhanlou, M. Ketabchi, N. Parvin, S. Khorsand, R. Bahrami, Accumulative press bonding; a novel manufacturing process of nanostructured metal matrix composites, Materials \& Design, 51 (2013) 367-374.

[15] S. Khoramkhorshid, M. Alizadeh, A.H. Taghvaei, S. Scudino, Microstructure and mechanical properties of Al-based metal matrix composites reinforced with Al84Gd6Ni7Co3 glassy particles produced by accumulative roll bonding, Materials \& Design, 90 (2016) 137-144. [16] M. Sharifitabar, A. Sarani, S. Khorshahian, M. Shafiee Afarani, Fabrication of 5052A1/Al2O3 nanoceramic particle reinforced composite via friction stir processing route, Materials \& Design, 32 (2011) 4164-4172. 
[17] M. Emamy, M. Khodadadi, A. Honarbakhsh Raouf, N. Nasiri, The influence of Ni addition and hot-extrusion on the microstructure and tensile properties of $\mathrm{Al}-15 \% \mathrm{Mg} 2 \mathrm{Si}$ composite, Materials \& Design, 46 (2013) 381-390.

[18] O. Scheibelhofer, M.O. Besenhard, M. Piller, J.G. Khinast, Comparing particle size distributions of an arbitrary shape, Powder Technology, 294 (2016) 134-145.

[19] J. Nafar Dastgerdi, G. Marquis, B. Anbarlooie, S. Sankaranarayanan, M. Gupta, Microstructure-sensitive investigation on the plastic deformation and damage initiation of amorphous particles reinforced composites, Composite Structures, 142 (2016) 130-139.

[20] L. Fan, S. Chen, C. Watson, ANNUAL REVIEW solids mixing, Industrial \& Engineering Chemistry, 62 (1970) 53-69.

[21] S. Li, Y. Su, X. Zhu, H. Jin, Q. Ouyang, D. Zhang, Enhanced mechanical behavior and fabrication of silicon carbide particles covered by in-situ carbon nanotube reinforced 6061 aluminum matrix composites, Materials \& Design, 107 (2016) 130-138.

[22] D. Hashiguchi, D. Tricker, A. Tarrant, J. Campbell, C. Pokross, Discontinuously reinforced aluminum MMC extrusions, Metal Powder Report, (2016).

[23] T. Clyne, P. Withers, An introduction to metal matrix composites, Cambridge University Press 1995.

[24] M. Tan, X. Zhang, Powder metal matrix composites: selection and processing, Materials Science and Engineering: A, 244 (1998) 80-85.

[25] O. Y1lmaz, S. Buytoz, Abrasive wear of Al2O3-reinforced aluminium-based MMCs, Composites Science and Technology, 61 (2001) 2381-2392.

[26] I. Ciftci, M. Turker, U. Seker, CBN cutting tool wear during machining of particulate reinforced MMCs, Wear, 257 (2004) 1041-1046.

[27] M. Jahedi, B. Mani, S. Shakoorian, E. Pourkhorshid, M.H. Paydar, Deformation rate effect on the microstructure and mechanical properties of $\mathrm{Al}-\mathrm{SiC} \mathrm{p}$ composites consolidated by hot extrusion, Materials Science and Engineering: A, 556 (2012) 23-30.

[28] H. Zare, M. Jahedi, M.R. Toroghinejad, M. Meratian, M. Knezevic, Microstructure and mechanical properties of carbon nanotubes reinforced aluminum matrix composites synthesized via equal-channel angular pressing, Materials Science and Engineering: A, 670 (2016) 205-216.

[29] V.V. Stolyarov, Y.T. Zhu, I.V. Alexandrov, T.C. Lowe, R.Z. Valiev, Influence of ECAP routes on the microstructure and properties of pure Ti, Materials Science and Engineering: A, 299 (2001) 59-67.

[30] M. Alizadeh, M. Paydar, D. Terada, N. Tsuji, Effect of SiC particles on the microstructure evolution and mechanical properties of aluminum during ARB process, Materials Science and Engineering: A, 540 (2012) 13-23.

[31] M. Knezevic, T. Nizolek, M. Ardeljan, I.J. Beyerlein, N.A. Mara, T.M. Pollock, Texture evolution in two-phase $\mathrm{Zr} / \mathrm{Nb}$ lamellar composites during accumulative roll bonding, International Journal of Plasticity, 57 (2014) 16-28.

[32] M. Ardeljan, M. Knezevic, T. Nizolek, I.J. Beyerlein, N.A. Mara, T.M. Pollock, A study of microstructure-driven strain localizations in two-phase polycrystalline $\mathrm{HCP} / \mathrm{BCC}$ composites using a multi-scale model, International Journal of Plasticity, 74 (2015) 35-57.

[33] I. Sabirov, O. Kolednik, R. Pippan, Homogenization of metal matrix composites by highpressure torsion, Metallurgical and Materials Transactions A, 36 (2005) 2861-2870.

[34] M. Jahedi, M.H. Paydar, S. Zheng, I.J. Beyerlein, M. Knezevic, Texture evolution and enhanced grain refinement under high-pressure-double-torsion, Materials Science and Engineering: A, 611 (2014) 29-36. 
[35] M. Jahedi, M. Knezevic, M.H. Paydar, High-pressure double torsion as a severe plastic deformation process: experimental procedure and finite element modeling, Journal of Materials Engineering and Performance, 24 (2015) 1471-1482.

[36] M. Jahedi, I.J. Beyerlein, M.H. Paydar, S. Zheng, T. Xiong, M. Knezevic, Effects of Pressure and Number of Turns on Microstructural Homogeneity Developed in High-Pressure Double Torsion, Metallurgical and Materials Transactions A, (2016) 1-15.

[37] M. Jahedi, M. Ardeljan, I.J. Beyerlein, M.H. Paydar, M. Knezevic, Enhancement of orientation gradients during simple shear deformation by application of simple compression, Journal of Applied Physics, 117 (2015) 214309.

[38] A.W. Nienow, M. Edwards, N. Harnby, Mixing in the process industries, ButterworthHeinemann1997.

[39] A. Rogers, Statistical analysis of spatial dispersion: the quadrat method, Pion 1974.

[40] P. Danckwerts, The definition and measurement of some characteristics of mixtures, Applied Scientific Research, Section A, 3 (1952) 279-296.

[41] P.M.C. Lacey, Developments in the theory of particle mixing, Journal of applied chemistry, 4 (1954) 257-268.

[42] J.G. Rosas, M. Blanco, A criterion for assessing homogeneity distribution in hyperspectral images. Part 1: homogeneity index bases and blending processes, Journal of pharmaceutical and biomedical analysis, 70 (2012) 680-690.

[43] G. Cairns, P. Goodwin, G. Wright, A decision-analysis-based framework for analysing stakeholder behaviour in scenario planning, European Journal of Operational Research, 249 (2016) 1050-1062.

[44] S.-J. Wang, S.-Y. Lee, Sensitivity analysis of structural equation models with equality functional constraints, Computational Statistics \& Data Analysis, 23 (1996) 239-256.

[45] M. Hadzima-Nyarko, E.K. Nyarko, D. Morić, A neural network based modelling and sensitivity analysis of damage ratio coefficient, Expert systems with applications, 38 (2011) 13405-13413.

[46] Y.-D. Ko, H. Shang, A neural network-based soft sensor for particle size distribution using image analysis, Powder Technology, 212 (2011) 359-366.

[47] E. Zio, N. Pedroni, Monte Carlo simulation-based sensitivity analysis of the model of a thermal-hydraulic passive system, Reliability Engineering \& System Safety, 107 (2012) 90-106. [48] Y. Zhang, H. Zhao, I. Hassinger, L.C. Brinson, L.S. Schadler, W. Chen, Microstructure reconstruction and structural equation modeling for computational design of nanodielectrics, Integrating Materials and Manufacturing Innovation, 4 (2015) 1-26.

[49] D.F. Millie, G.R. Weckman, G.L. Fahnenstiel, H.J. Carrick, E. Ardjmand, W.A. Young, M.J. Sayers, R.A. Shuchman, Using artificial intelligence for CyanoHAB niche modeling: discovery and visualization of Microcystis-environmental associations within western Lake Erie, Canadian Journal of Fisheries and Aquatic Sciences, 71 (2014) 1642-1654.

[50] D.F. Millie, G.R. Weckman, W.A. Young, J.E. Ivey, D.P. Fries, E. Ardjmand, G.L. Fahnenstiel, Coastal 'Big Data'and nature-inspired computation: Prediction potentials, uncertainties, and knowledge derivation of neural networks for an algal metric, Estuarine, Coastal and Shelf Science, 125 (2013) 57-67.

[51] S. Malinov, W. Sha, Software products for modelling and simulation in materials science, Computational Materials Science, 28 (2003) 179-198.

[52] A. Doucet, N. De Freitas, N. Gordon, An introduction to sequential Monte Carlo methods, Sequential Monte Carlo methods in practice, Springer2001, pp. 3-14. 
[53] M. Altan, Reducing shrinkage in injection moldings via the Taguchi, ANOVA and neural network methods, Materials \& Design, 31 (2010) 599-604.

[54] B. Balta, A.A. Arici, M. Yilmaz, Optimization of process parameters for friction weld steel tube to forging joints, Materials \& Design, 103 (2016) 209-222.

[55] A.R. Kennedy, S.M. Wyatt, The effect of processing on the mechanical properties and interfacial strength of aluminium/TiC MMCs, Composites Science and Technology, 60 (2000) 307-314.

[56] D.J. Lloyd, Aspects of fracture in particulate reinforced metal matrix composites, Acta Metallurgica et Materialia, 39 (1991) 59-71.

[57] J.D. Boyd, D.J. Lloyd, 3.06 - Clustering in Particulate MMCs A2 - Kelly, Anthony, in: C. Zweben (Ed.) Comprehensive Composite Materials, Pergamon, Oxford, 2000, pp. 139-149.

[58] A.F. Whitehouse, H.M.A. Winand, T.W. Clyne, The effect of processing route and reinforcement geometry on isothermal creep behaviour of particulate and short fibre MMCs, Materials Science and Engineering: A, 242 (1998) 57-69.

[59] Y. Yang, J. Lan, X. Li, Study on bulk aluminum matrix nano-composite fabricated by ultrasonic dispersion of nano-sized $\mathrm{SiC}$ particles in molten aluminum alloy, Materials Science and Engineering: A, 380 (2004) 378-383.

[60] J. Corrochano, M. Lieblich, J. Ibáñez, On the role of matrix grain size and particulate reinforcement on the hardness of powder metallurgy $\mathrm{Al}-\mathrm{Mg}-\mathrm{Si} / \mathrm{MoSi} 2$ composites, Composites Science and Technology, 69 (2009) 1818-1824.

[61] O. El-Kady, A. Fathy, Effect of SiC particle size on the physical and mechanical properties of extruded Al matrix nanocomposites, Materials \& Design (1980-2015), 54 (2014) 348-353.

[62] S. Madeira, G. Miranda, V.H. Carneiro, D. Soares, F.S. Silva, O. Carvalho, The effect of SiCp size on high temperature damping capacity and dynamic Young's modulus of hot-pressed AlSi-SiCp MMCs, Materials \& Design, 93 (2016) 409-417.

[63] Q. Zhang, D.L. Chen, A model for predicting the particle size dependence of the low cycle fatigue life in discontinuously reinforced MMCs, Scripta Materialia, 51 (2004) 863-867.

[64] J.A. Hartigan, M.A. Wong, Algorithm AS 136: A k-means clustering algorithm, Journal of the Royal Statistical Society. Series C (Applied Statistics), 28 (1979) 100-108.

[65] T. Kanungo, D.M. Mount, N.S. Netanyahu, C.D. Piatko, R. Silverman, A.Y. Wu, An efficient k-means clustering algorithm: Analysis and implementation, Pattern Analysis and Machine Intelligence, IEEE Transactions on, 24 (2002) 881-892.

[66] P.S. Bradley, U.M. Fayyad, Refining Initial Points for K-Means Clustering, ICML, Citeseer, 1998, pp. 91-99.

[67] A. Likas, N. Vlassis, J.J. Verbeek, The global k-means clustering algorithm, Pattern recognition, 36 (2003) 451-461.

[68] R.C. Team, R: A language and environment for statistical computing, (2013).

[69] K. Poole, R. Taylor, G. Wall, Mixing powders to fine-scale homogeneity: studies of batch mixing, (1964).

[70] P.A. Karnezis, G. Durrant, B. Cantor, Characterization of Reinforcement Distribution in Cast Al-Alloy/SiCp Composites, Materials Characterization, 40 (1998) 97-109.

[71] J. Mousavi, M. Parvini, A sensitivity analysis of parameters affecting the hydrogen release and dispersion using ANOVA method, International Journal of Hydrogen Energy, 41 (2016) 5188-5201.

[72] A. Haber, R. Runyon, General Statistics. Addison, Wesiey Publishing Company, Inc., Reading, MA, 1977. 
[73] D.C. Montgomery, Design and analysis of experiments, John Wiley \& Sons2008.

[74] B.J. Winer, D.R. Brown, K.M. Michels, Statistical principles in experimental design, McGraw-Hill New York1971.

[75] C. Camposeco-Negrete, Optimization of cutting parameters for minimizing energy consumption in turning of AISI 6061 T6 using Taguchi methodology and ANOVA, Journal of Cleaner Production, 53 (2013) 195-203.

\section{Figures and tables captions:}

Table 1 Average $H$-metric values for HPDT and HPT processes at different locations and number of turns. The corresponding $P$-values based on the ANOVA test are also included in the table.

Table $2 P$-values based on the ANOVA test for HPDT 2 turn and HPT 4 turn processes.

Figure 1 SEM micrographs showing microstructure at middle and edge locations in MMC samples processed using (a) HPDT and (b) HPT to 2 and 6 number of turns.

Figure 2 SEM micrographs showing the cracks and porosities in the microstructure as a result of particle fragmentation.

Figure 3 SEM micrographs showing instances of fragmentation of $\mathrm{SiC}$ particles within $\mathrm{Cu}$ matrix during HPT and HPDT as a function of torsional turns. Arrows indicate cracks.

Figure 4 SEM micrographs showing instances of $\mathrm{SiC}$ particle clusters shortly after fragmentation during HPT and HPDT as a function of torsional turns. Arrows indicate these types of clusters.

Figure 5 A greyscale image (a) and the distribution of pixel greyscale values (b) in the image.

Figure 6 Effect of macro-pixel edge size (indicated in white box at the bottom) on tracking clusters. The arrows on the original micrograph show examples of clustered regions.

Figure 7 Schematic showing the statistical procedure for calculating the $H$-metric. 
Figure 8 Schematic of statistical analyses of a micrograph and a random image showing the distribution of particles in macro-pixels ( 0 and 1 correspond to black and white colors, respectively) and mean and standard deviation ( $\mathrm{Sd}$ ) values for 4 macro-pixels and whole images in both instances.

Figure $9 \mathrm{H}$-metric values for the HPDT process (a) as a function of turns at different locations and (b) at constant turn from center to edge.

Figure $10 \mathrm{SiC}$ particle size distributions for samples synthesized using the HPDT and HPT processes to different number of turns. The results are shown for three locations on the specimen as indicated in the legend.

Figure 11 Cumulative distribution functions (CDFs) of SiC particle size distributions at different sample locations and strain levels of HPT and HPDT processes.

Figure $12 \mathrm{SiC}$ particle size distributions at different sample locations for 4-turn HPT and 2-turn HPDT. 
\# of

turns

Middle

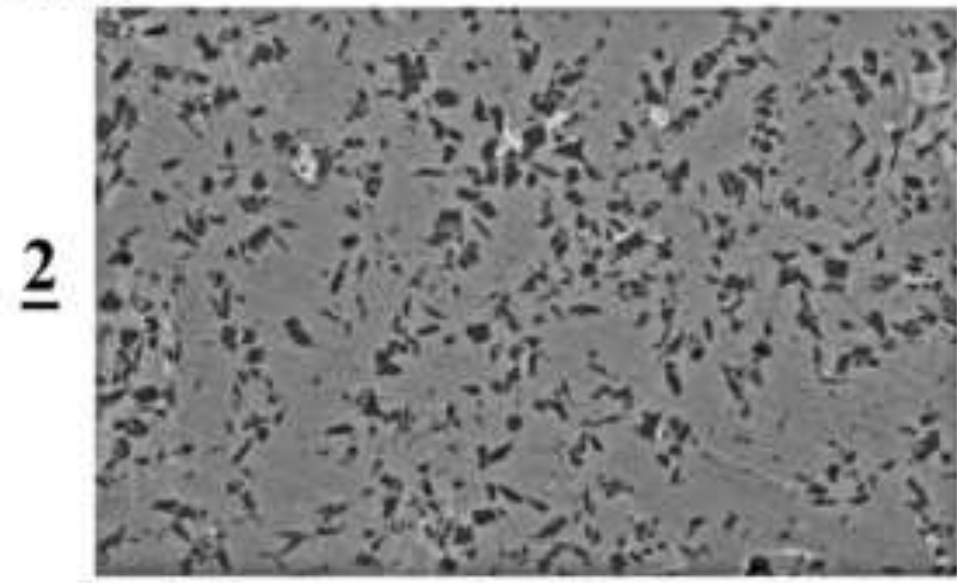

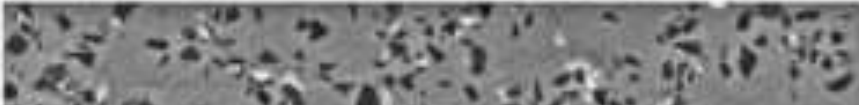

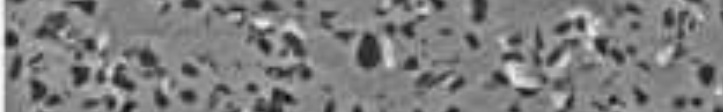

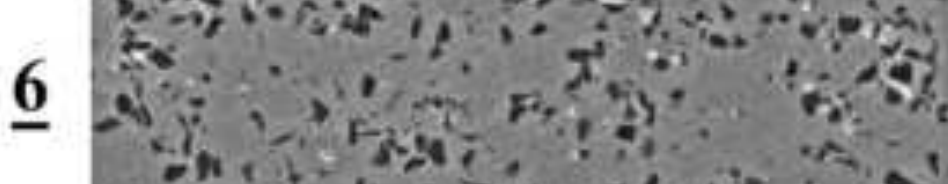

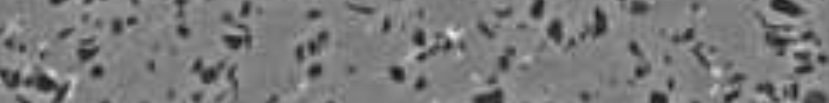

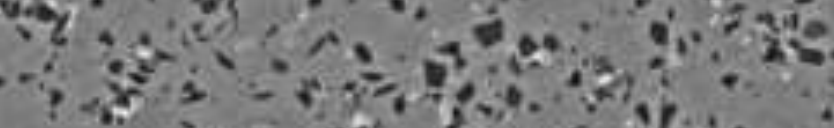

(a)

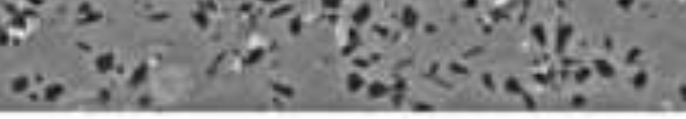
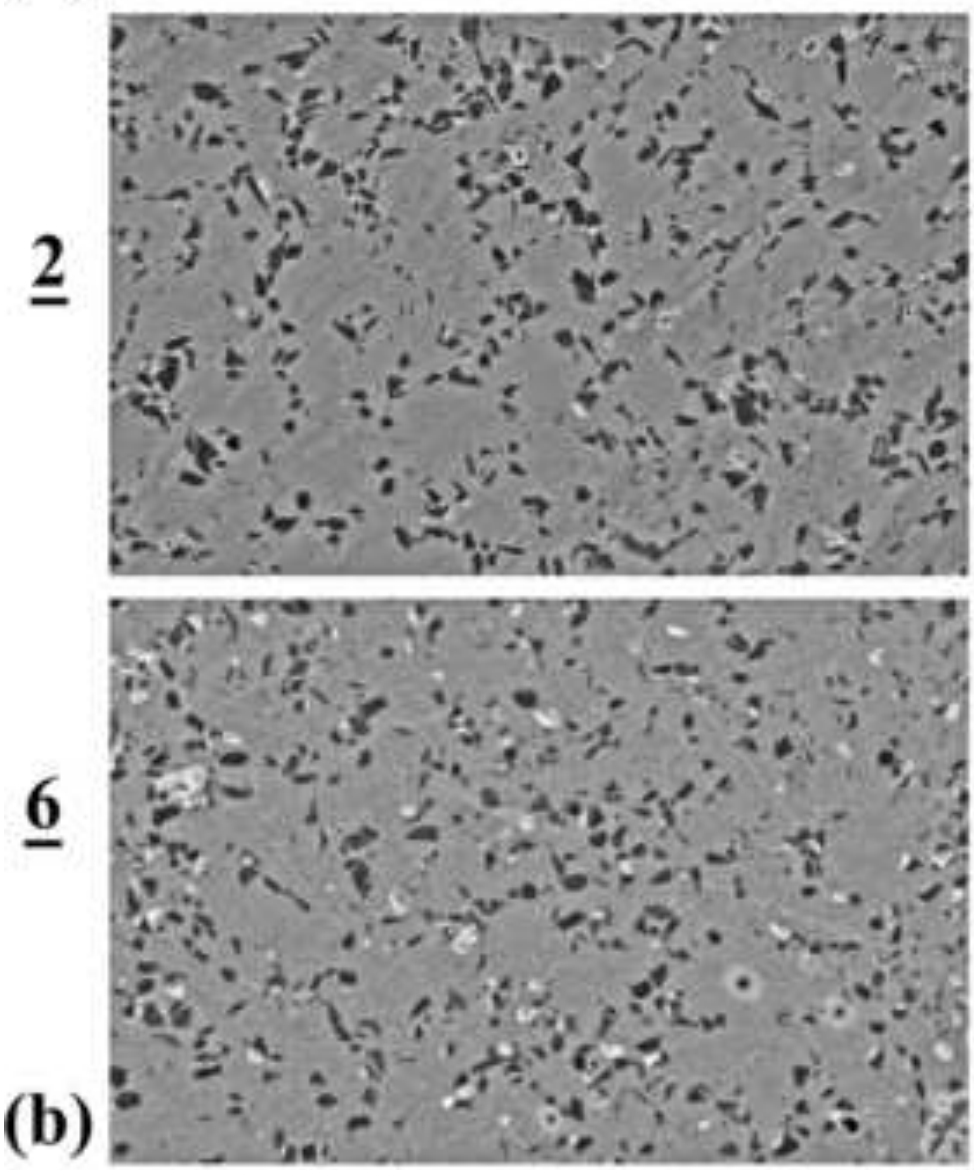

Edge

$30 \mu \mathrm{m}$

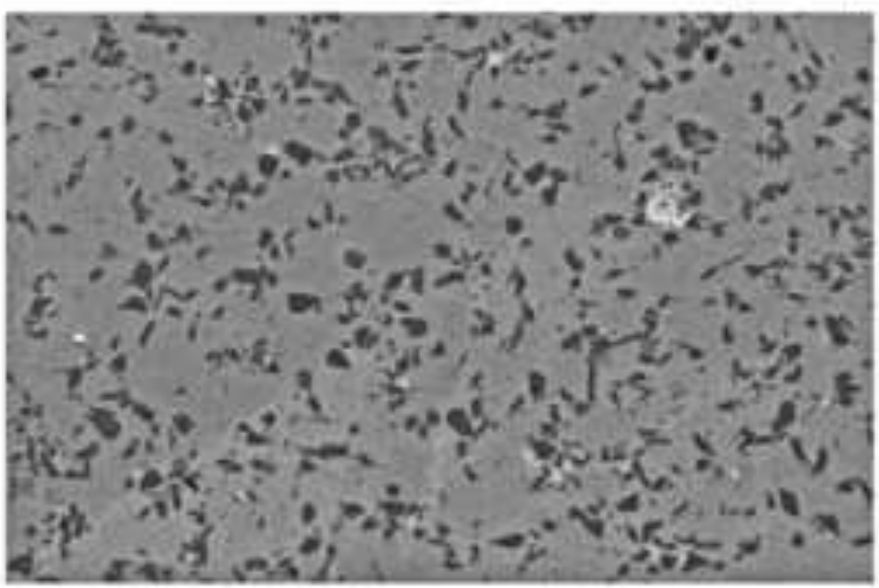

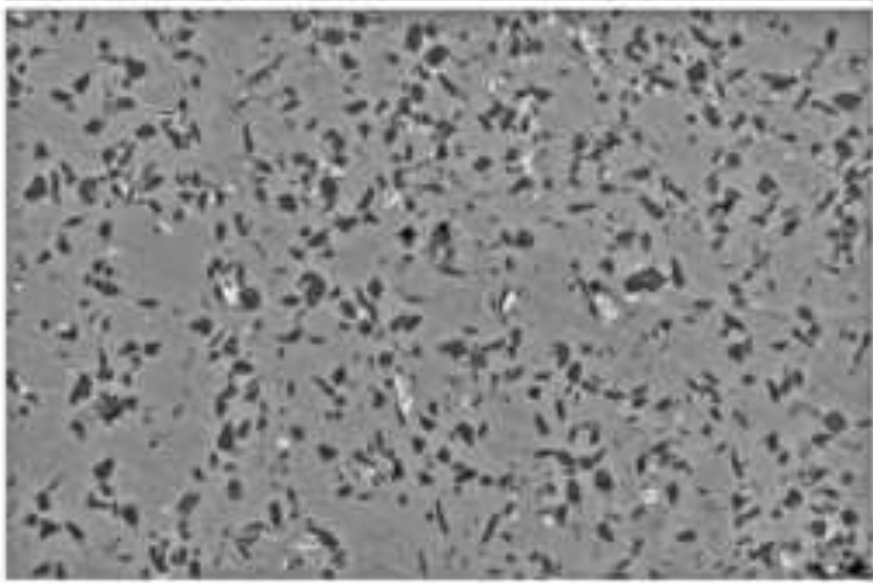
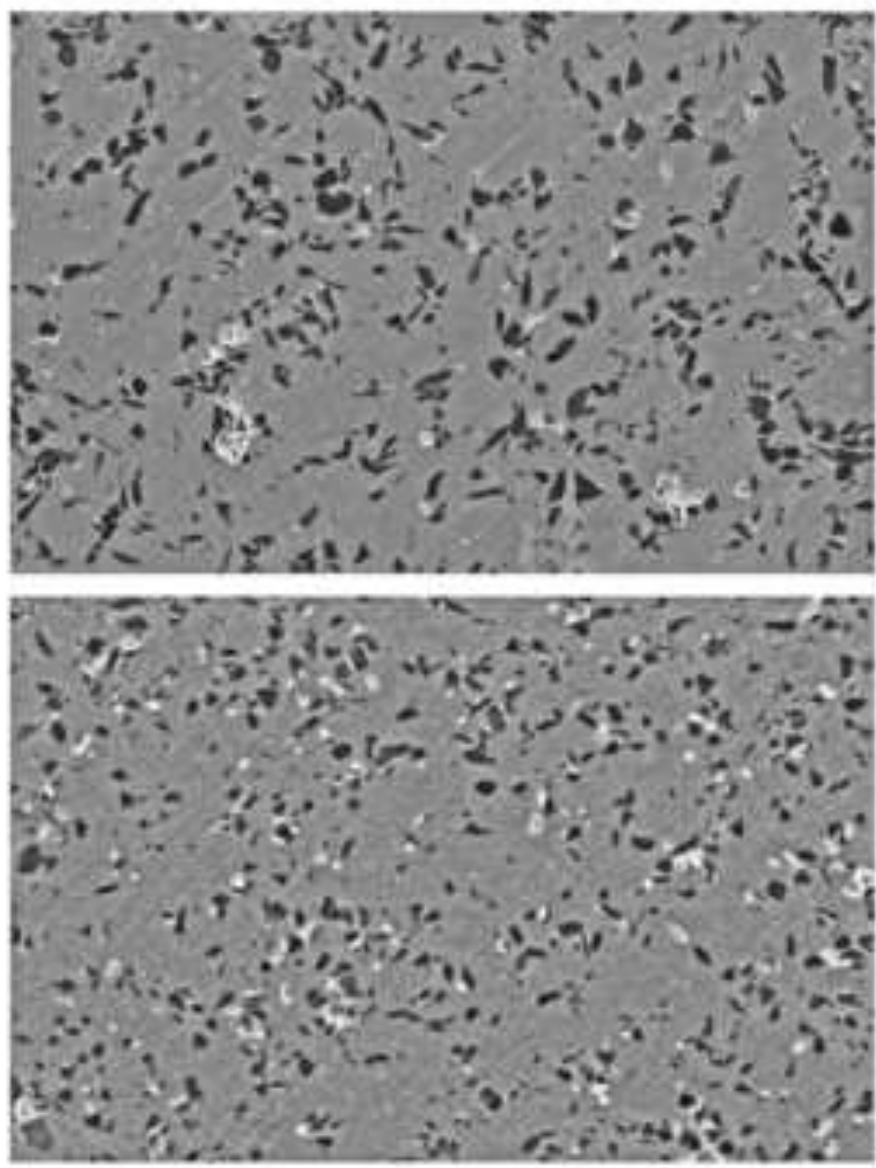

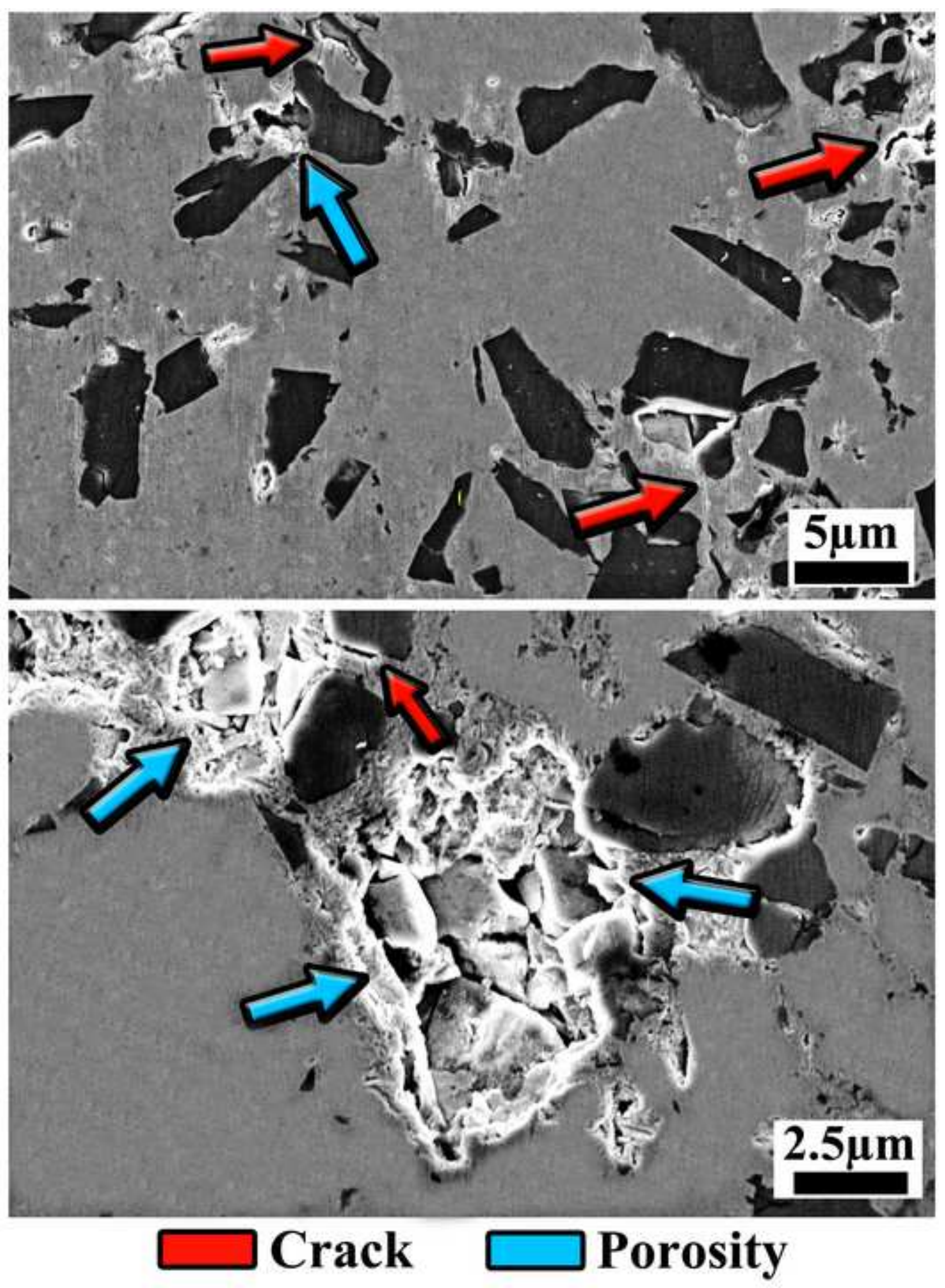

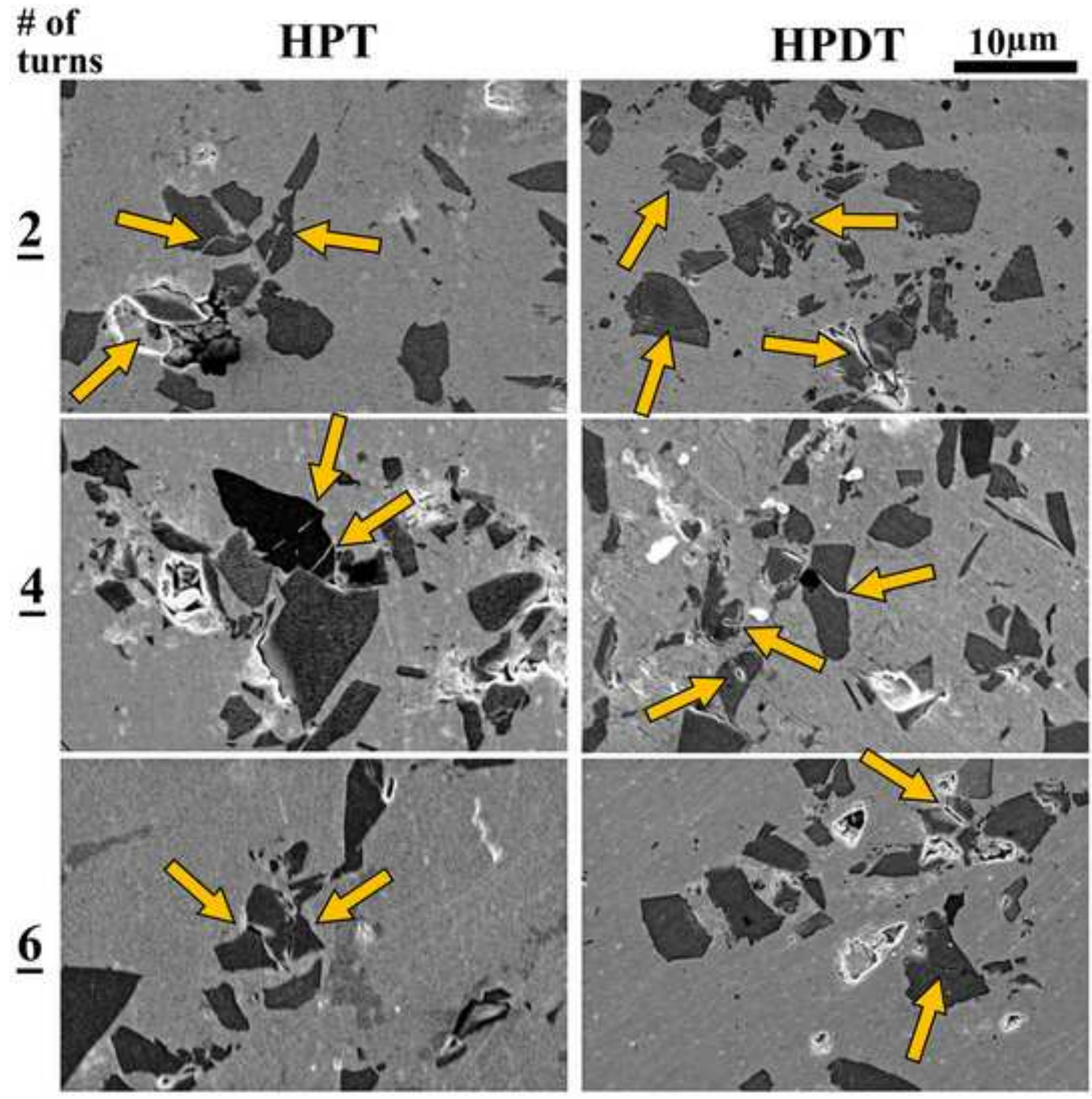

\section{HPDT $10 \mu \mathrm{m}$}



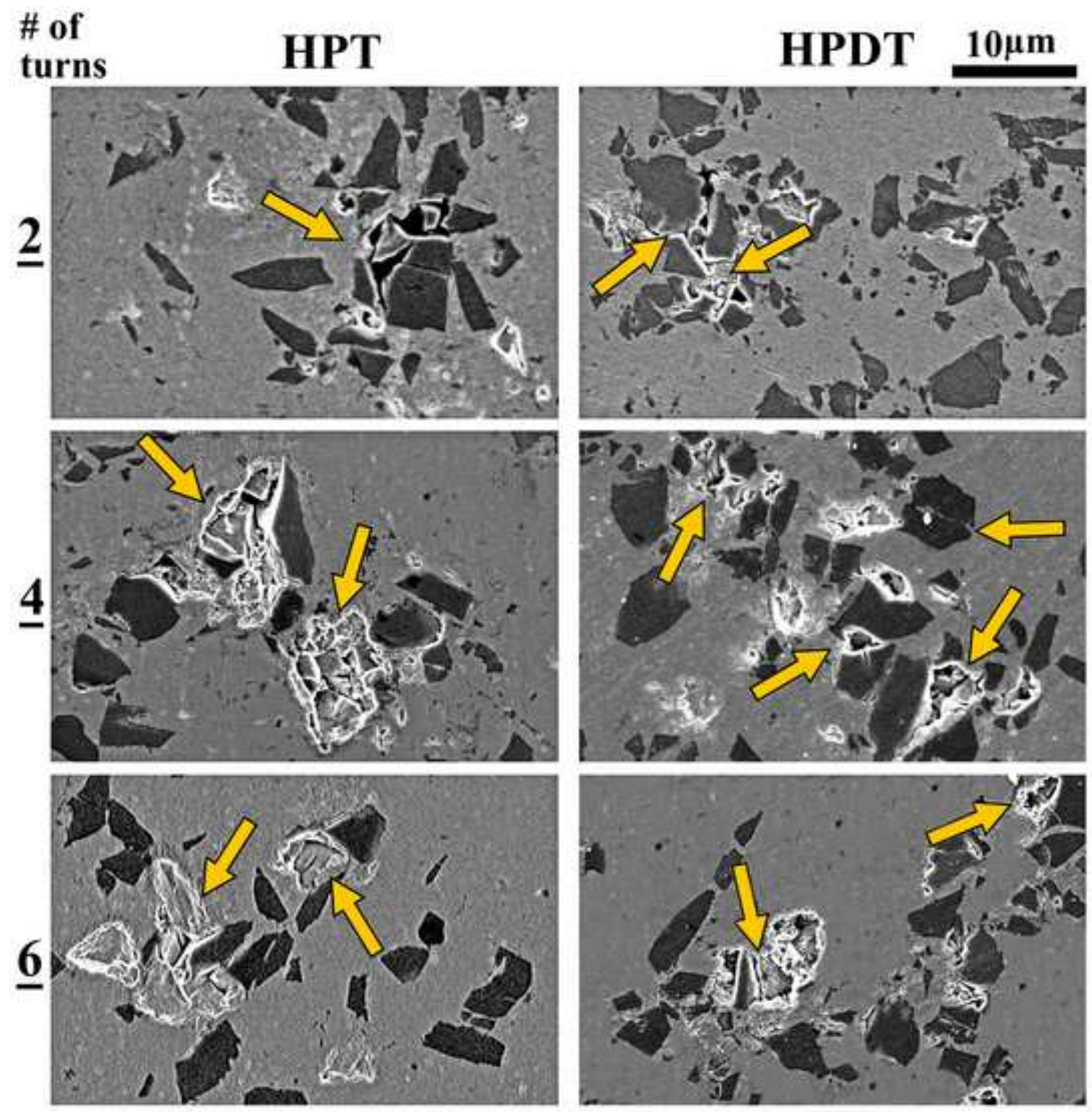

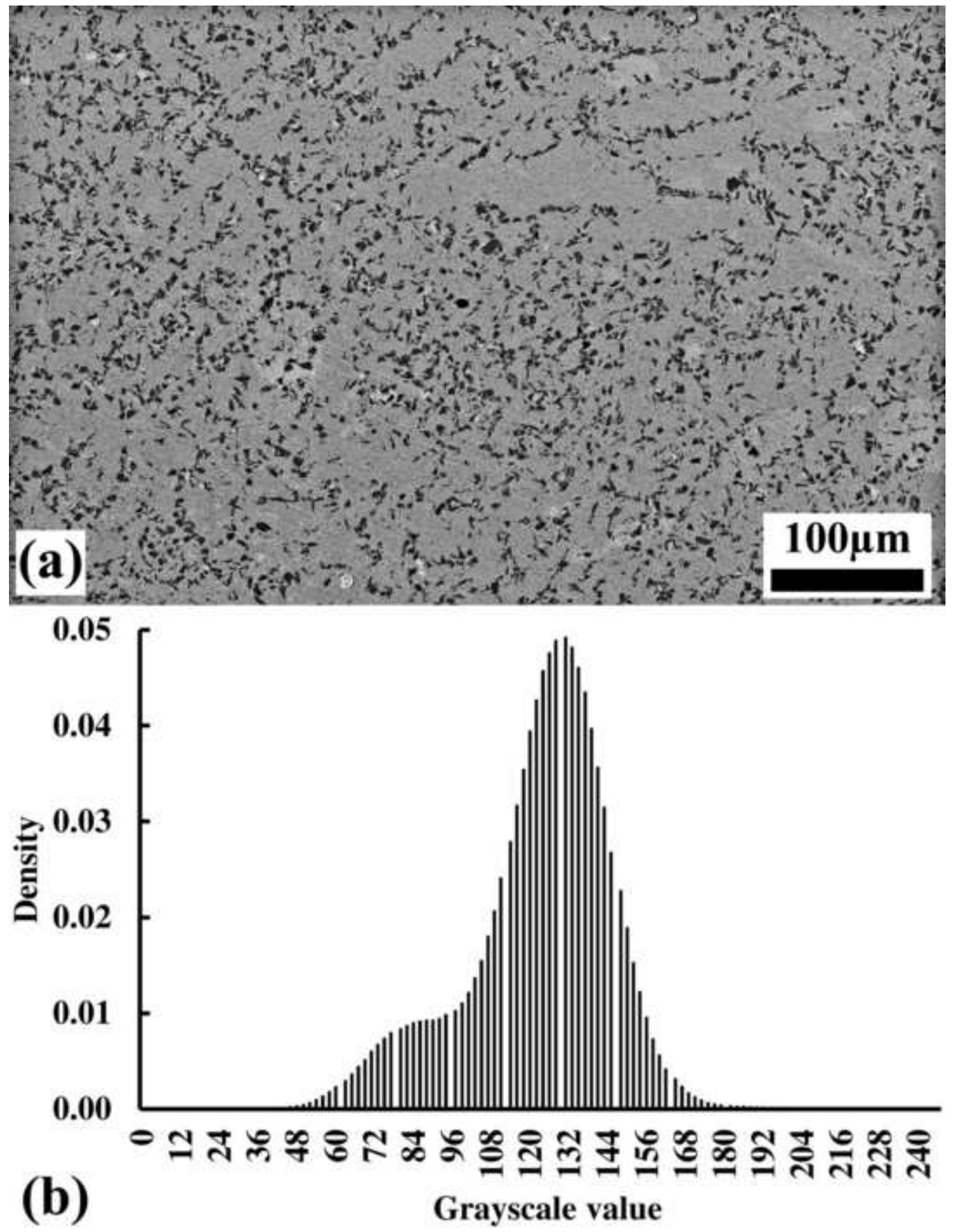

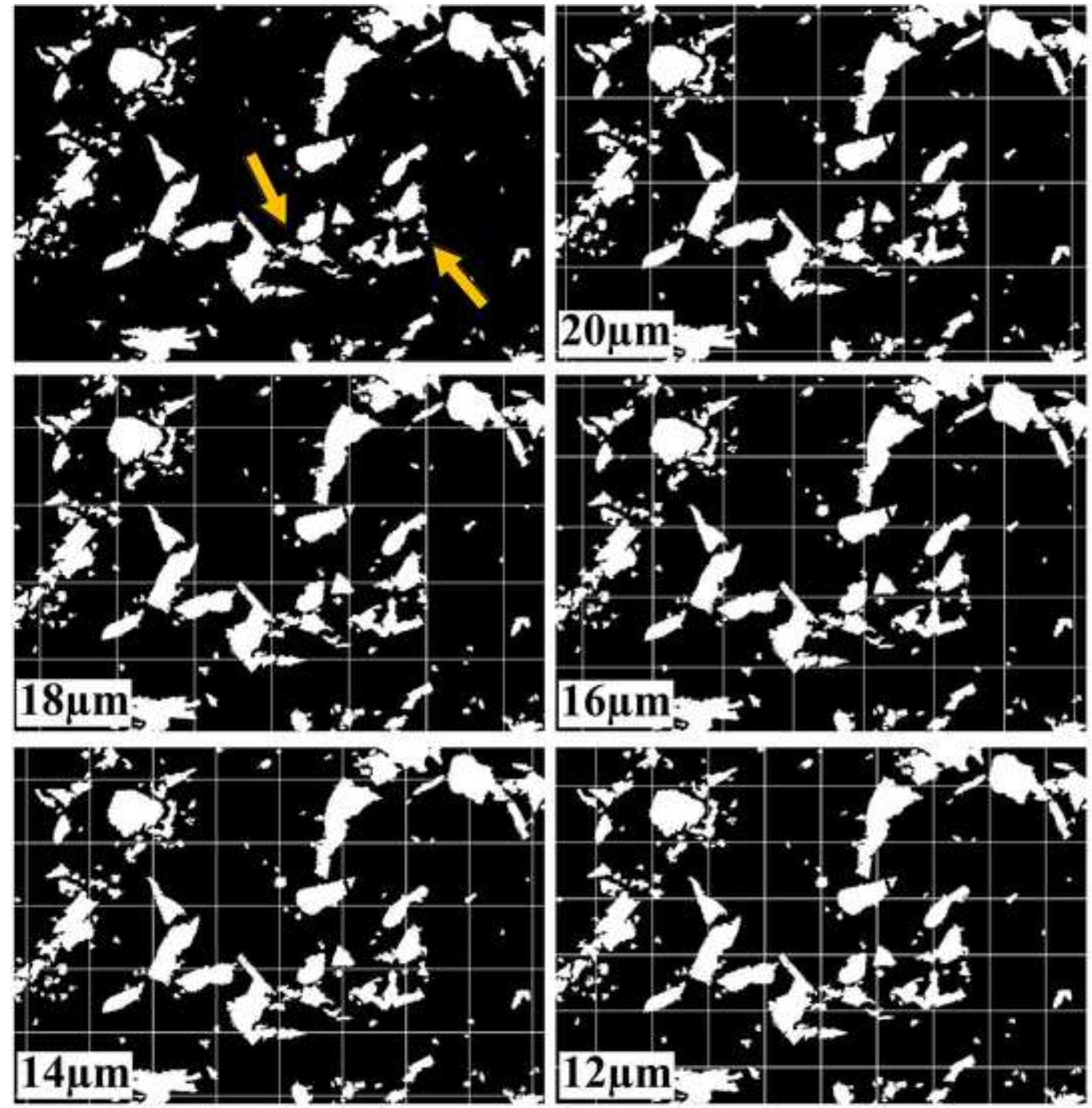


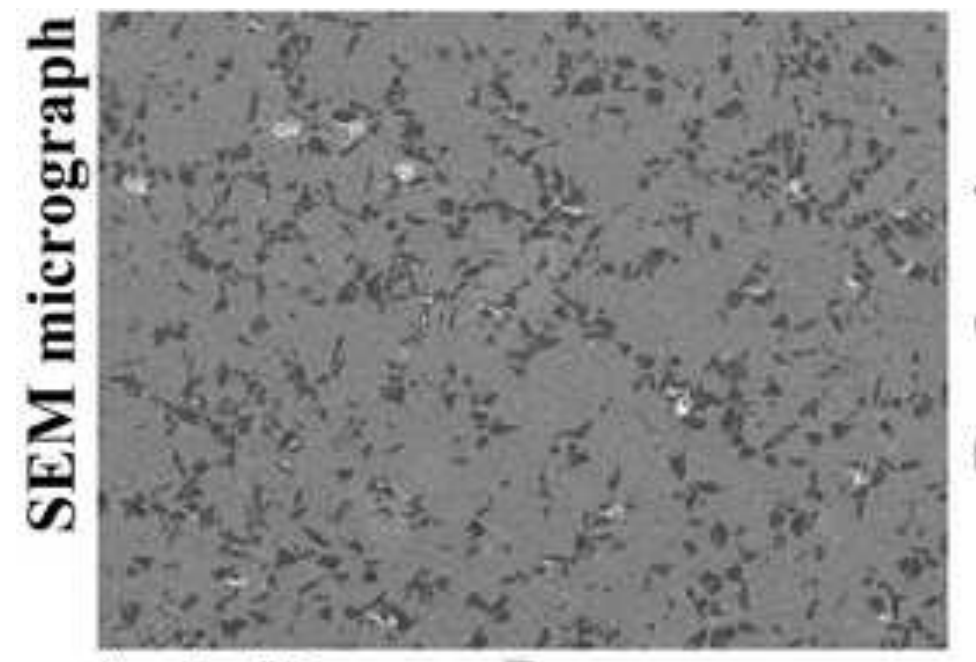

Apply $K$-means algorithm to find the threshold

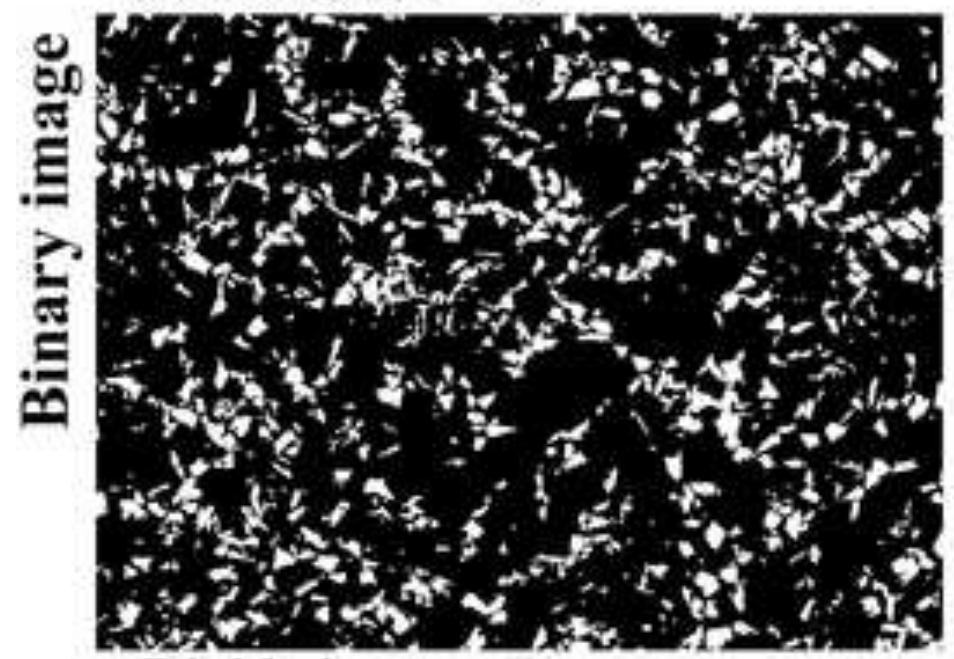

Divide image into macro-pixels

\begin{tabular}{|c|c|c}
\hline $\operatorname{Grid}_{1}\left(G_{1}\right)$ & $\operatorname{Grid}_{2}\left(G_{2}\right)$ & \\
$G_{S d_{1}}=S d$ in $G_{1}$ & $G_{S d_{2}}=S d$ in $G_{2}$ & $-\rightarrow$ \\
\hdashline $\operatorname{Grid}_{3}\left(G_{3}\right)$ & $\operatorname{Grid}_{4}\left(G_{4}\right)$ & \\
$G_{S d_{3}}=S d$ in $G_{3}$ & $G_{S d_{4}}=S d$ in $G_{4}$ & $-\rightarrow$ \\
\hdashline $\mathbf{I}$ & $\mathbf{I}$ & $\checkmark$ \\
$\mathbf{V}$ & $\mathbf{V}$ & $\mathbf{U}$
\end{tabular}

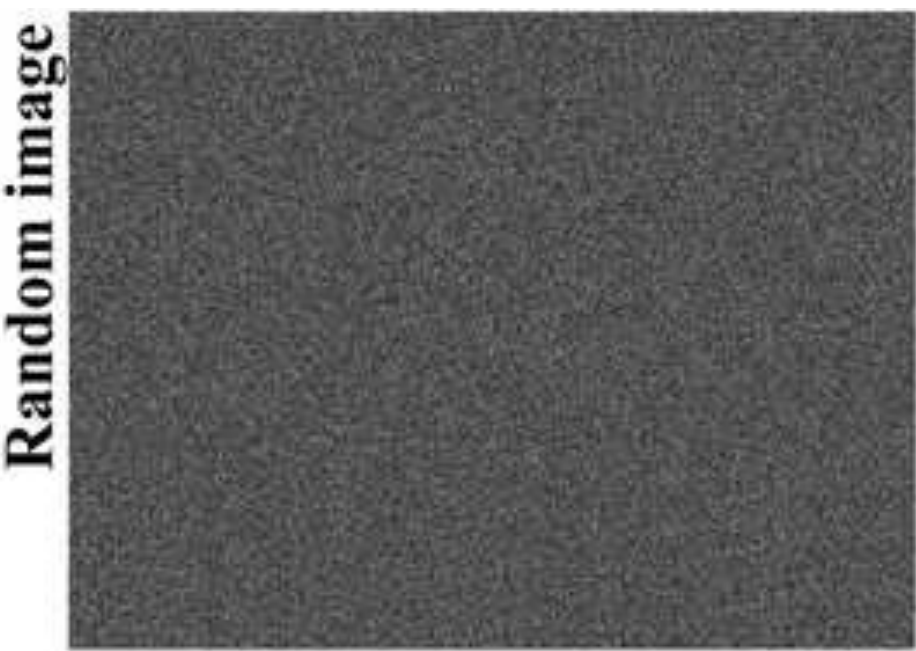

\section{Calculate average standard deviation of macro-pixels for random image}

\section{$\bar{G}_{S d \_r a n d o m}$}

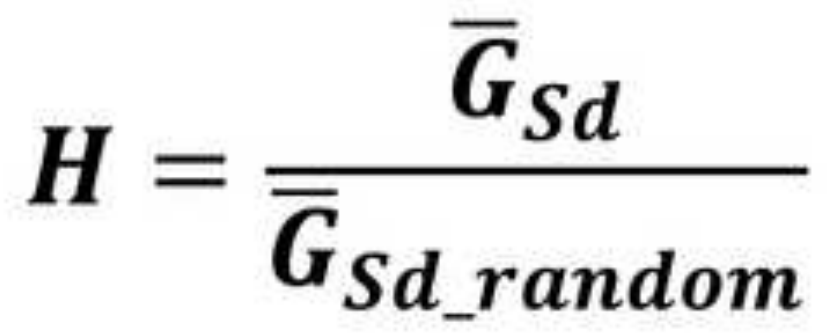

$\overline{\boldsymbol{G}}$

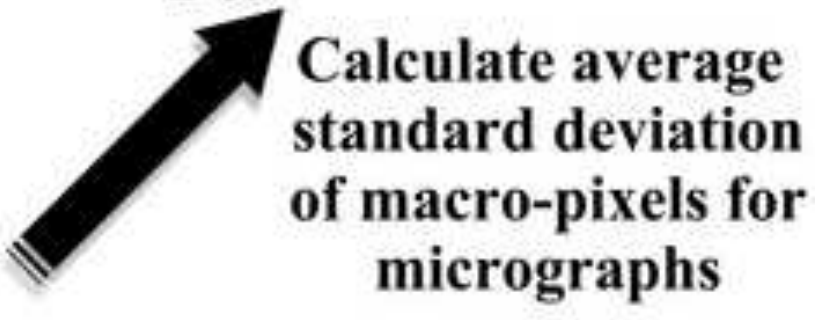

These sequences were repeated for 10 micrographs of each location. 

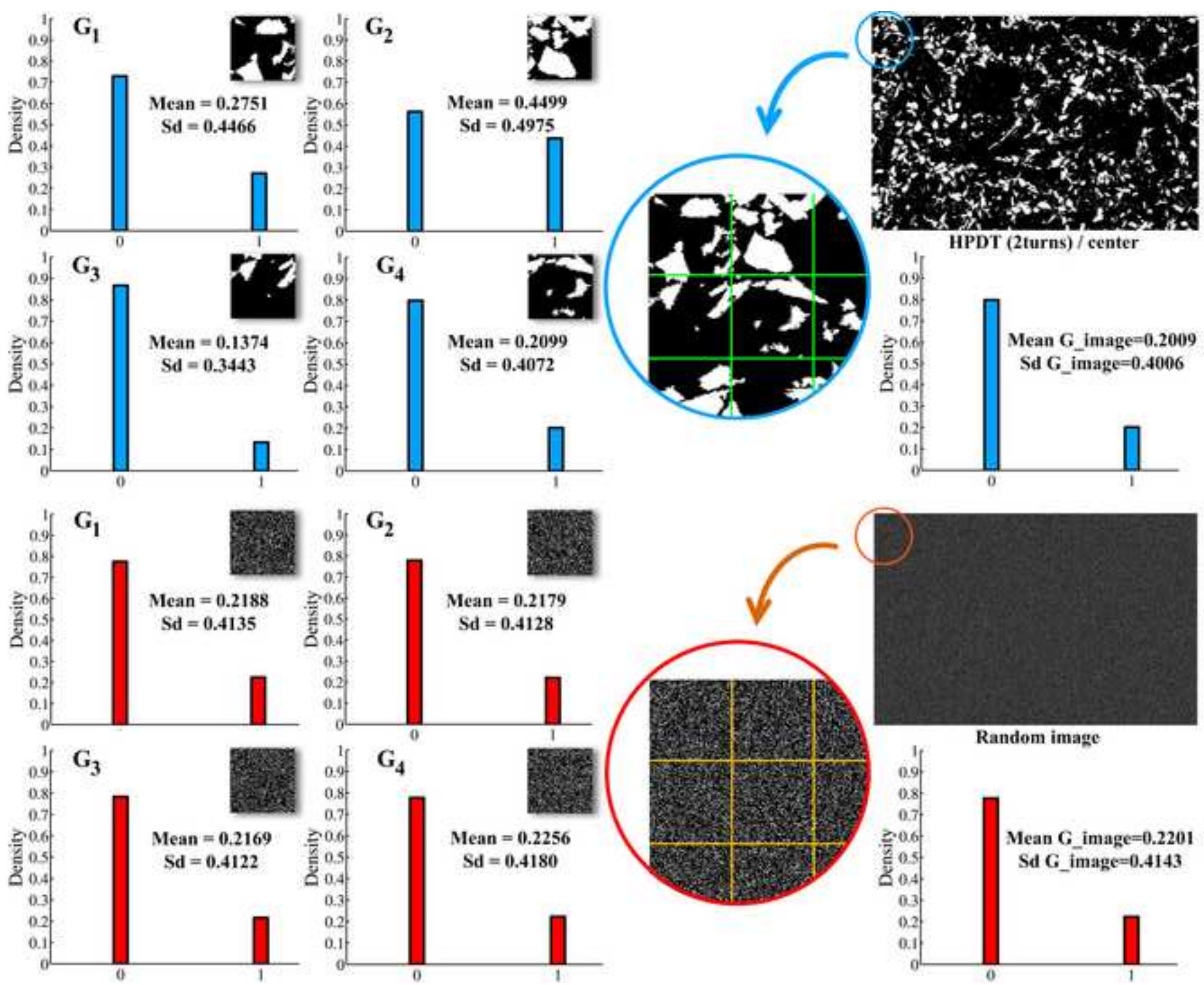

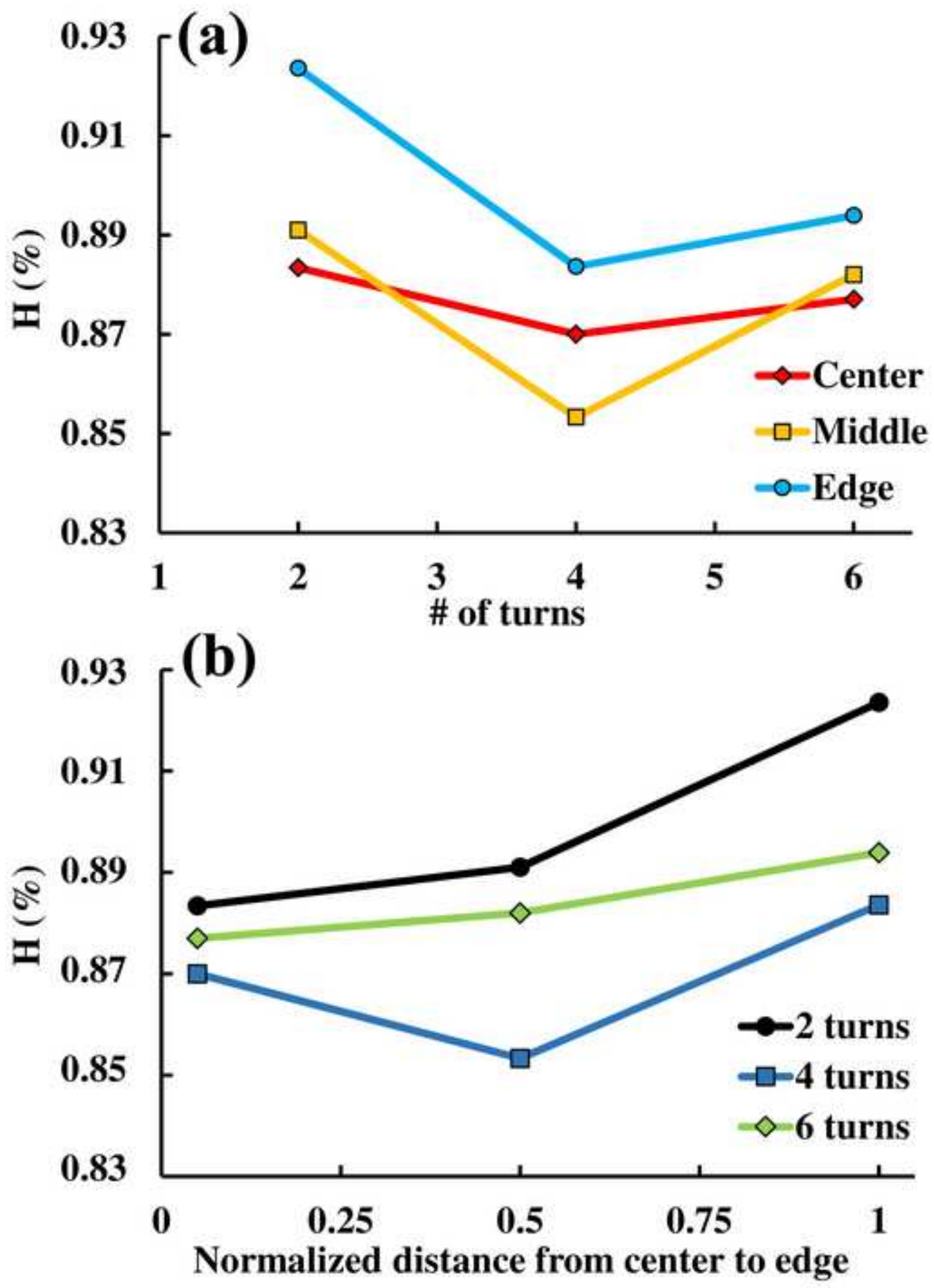

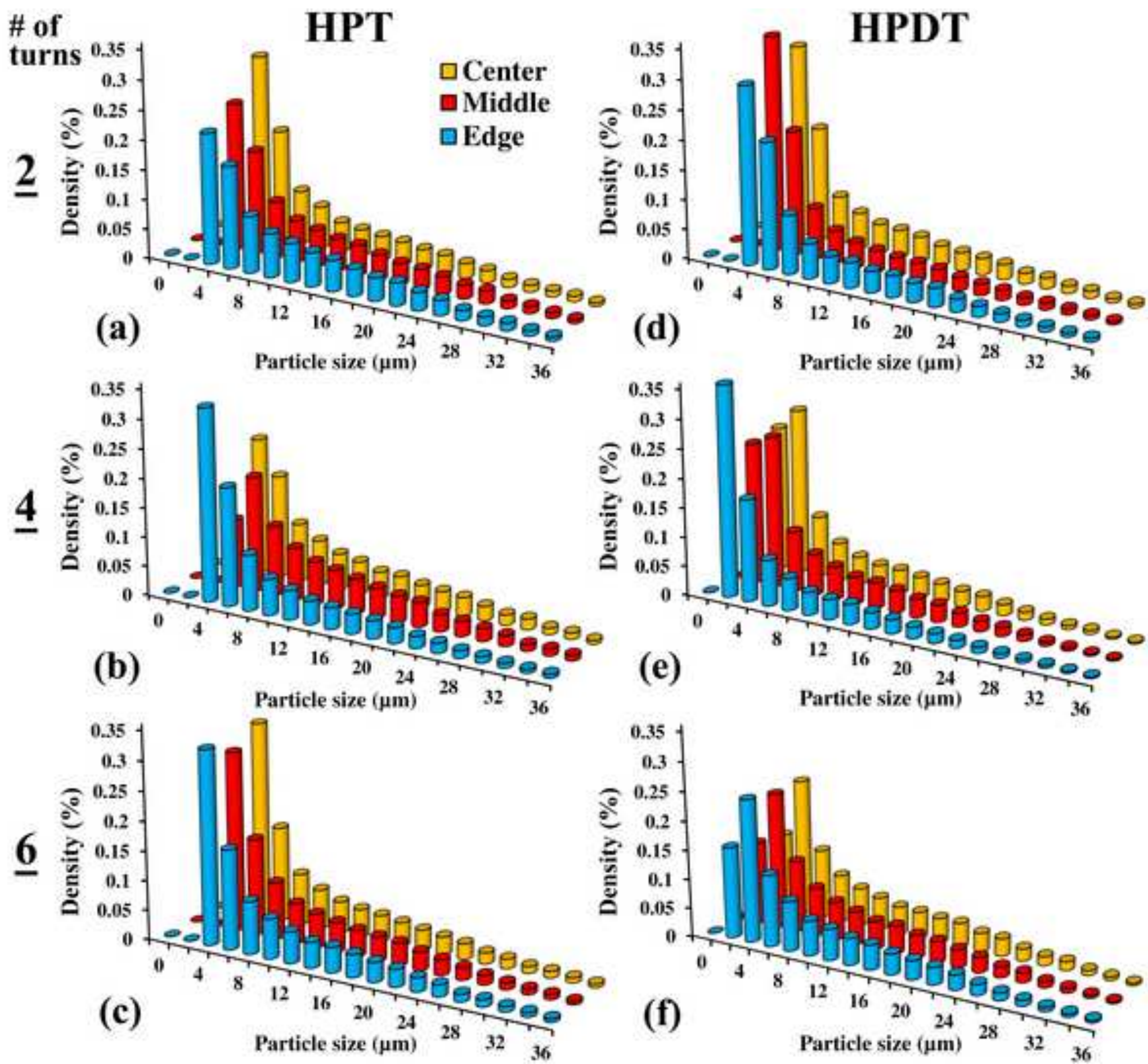


\section{\# of}
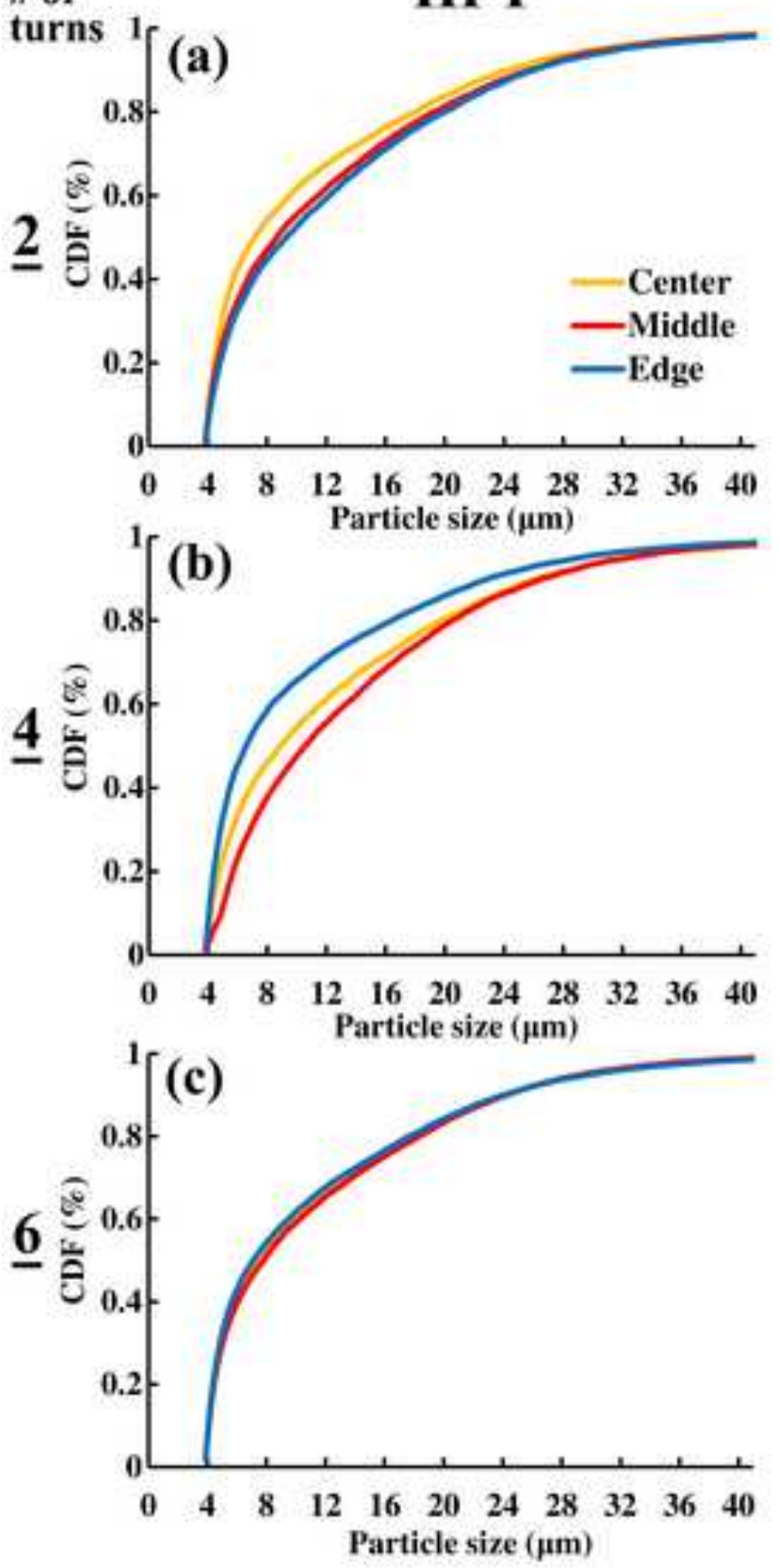

HPDT
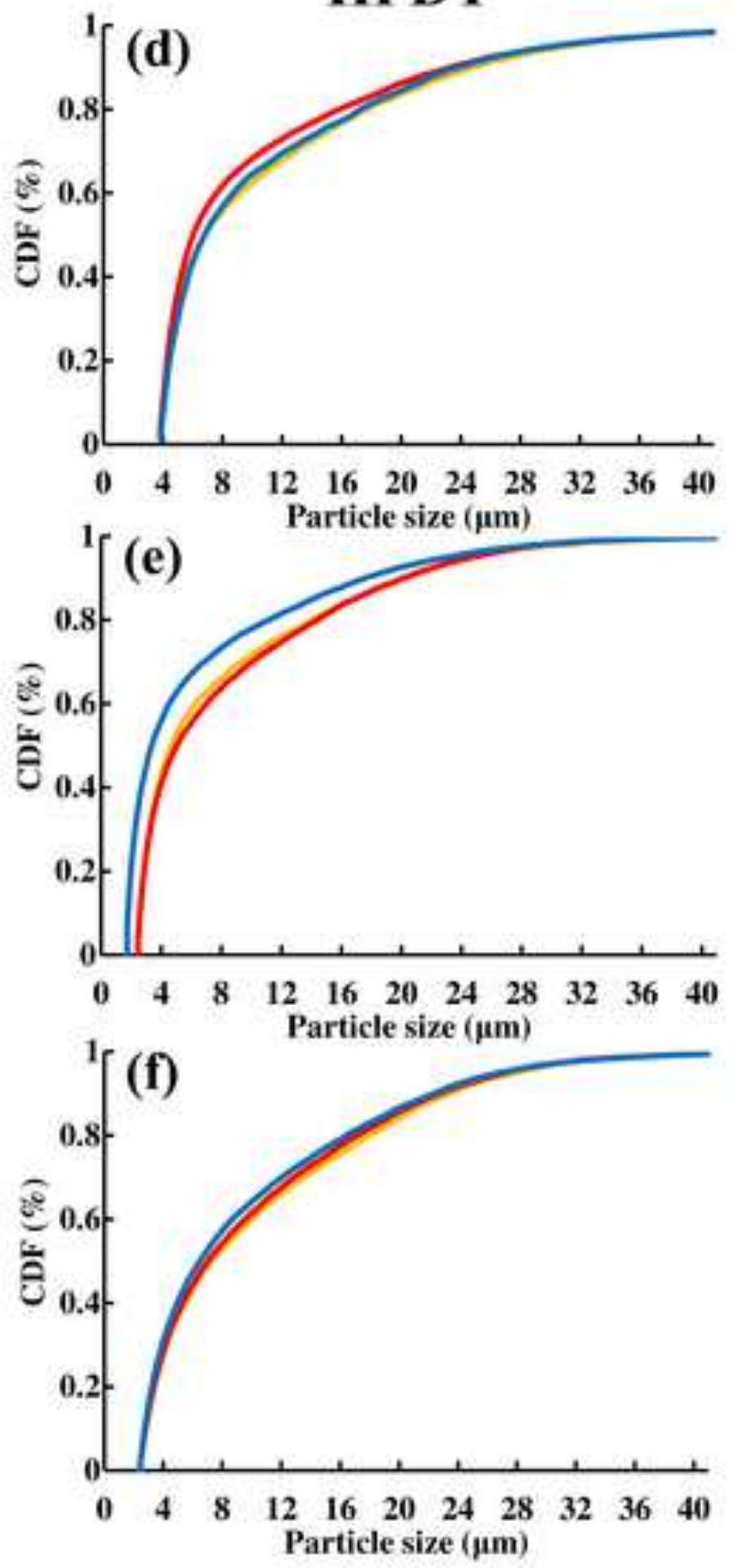


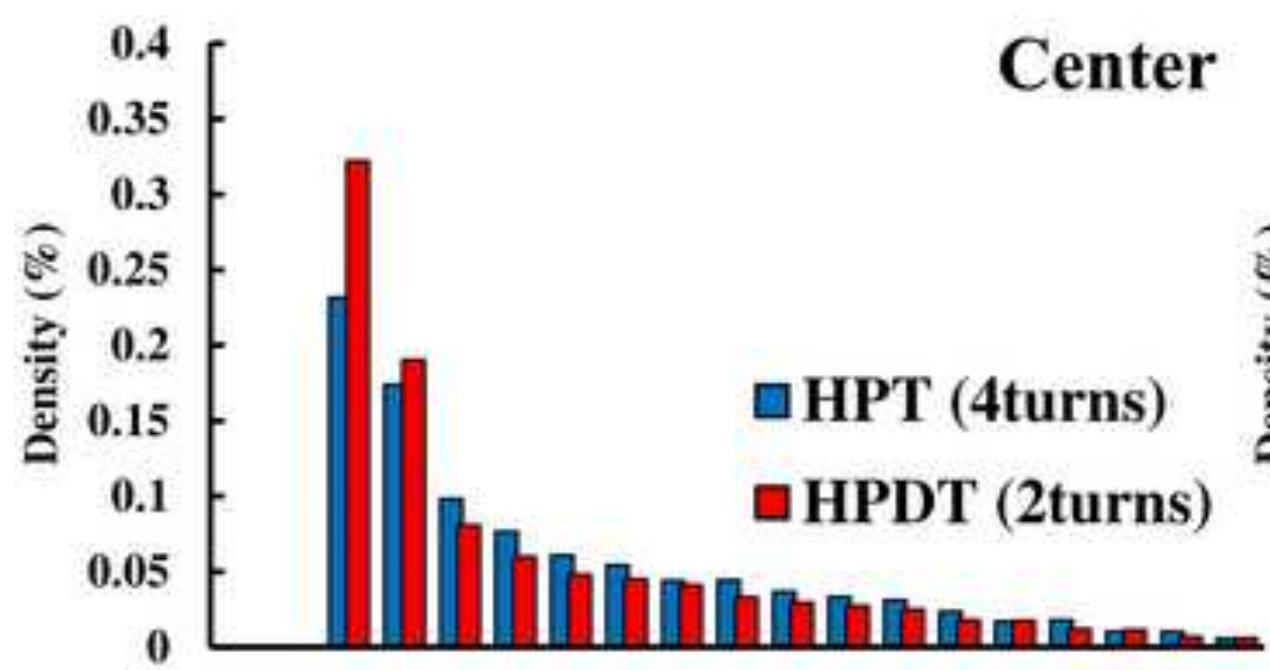

$0 \begin{array}{lllllll} & 2 & 4 & 8 & 1012141618202224262830323436\end{array}$

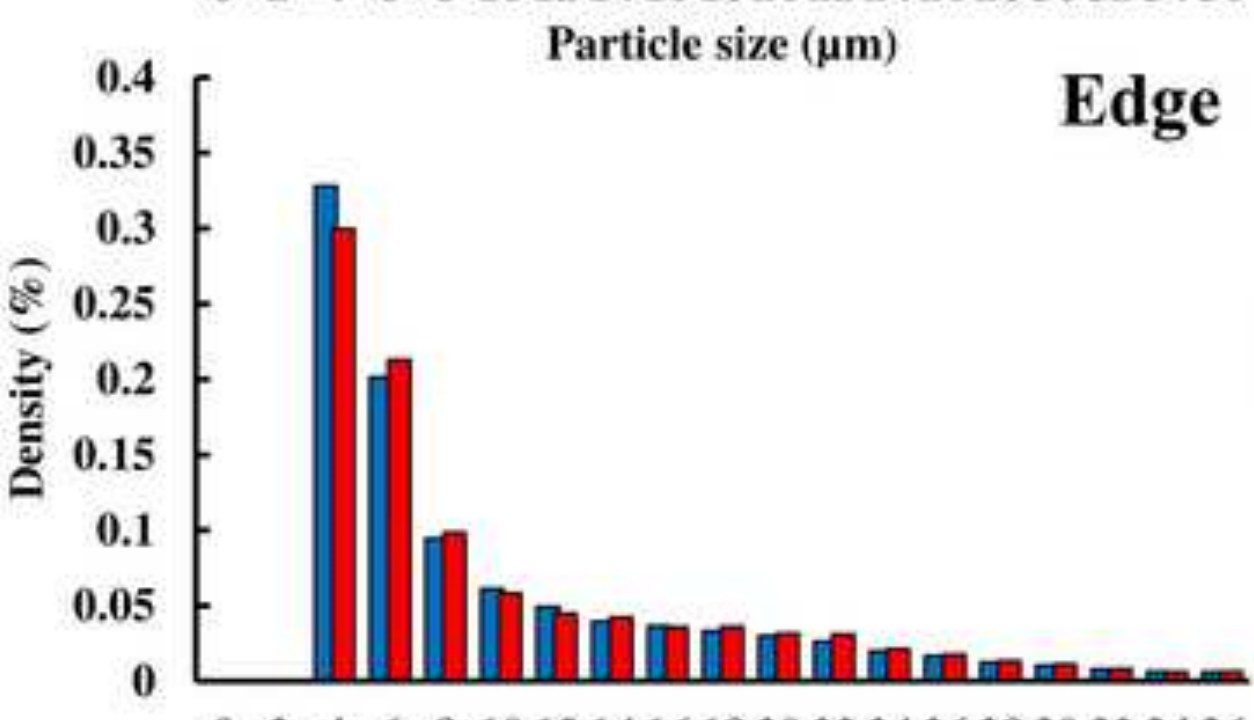

0224681012141618202224262830323436

Particle size $(\mu \mathrm{m})$

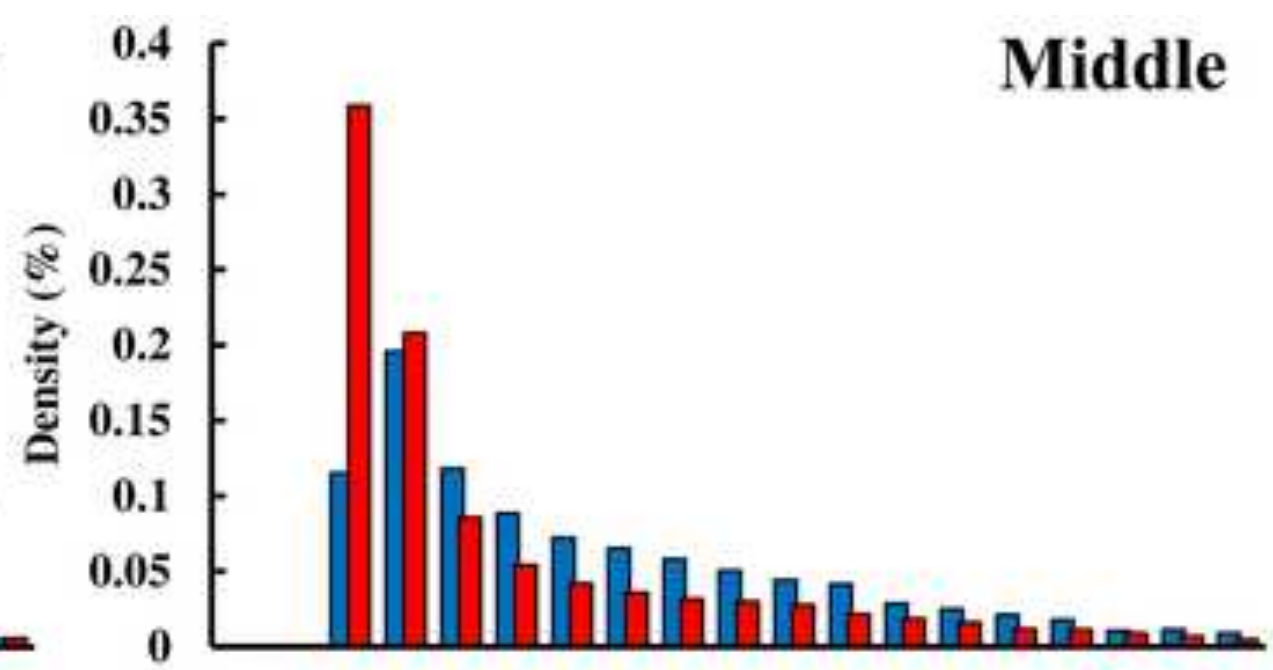

$\begin{array}{lllllll}0 & 2 & 4 & 6 & 8 & 1012141618202224262830323436\end{array}$

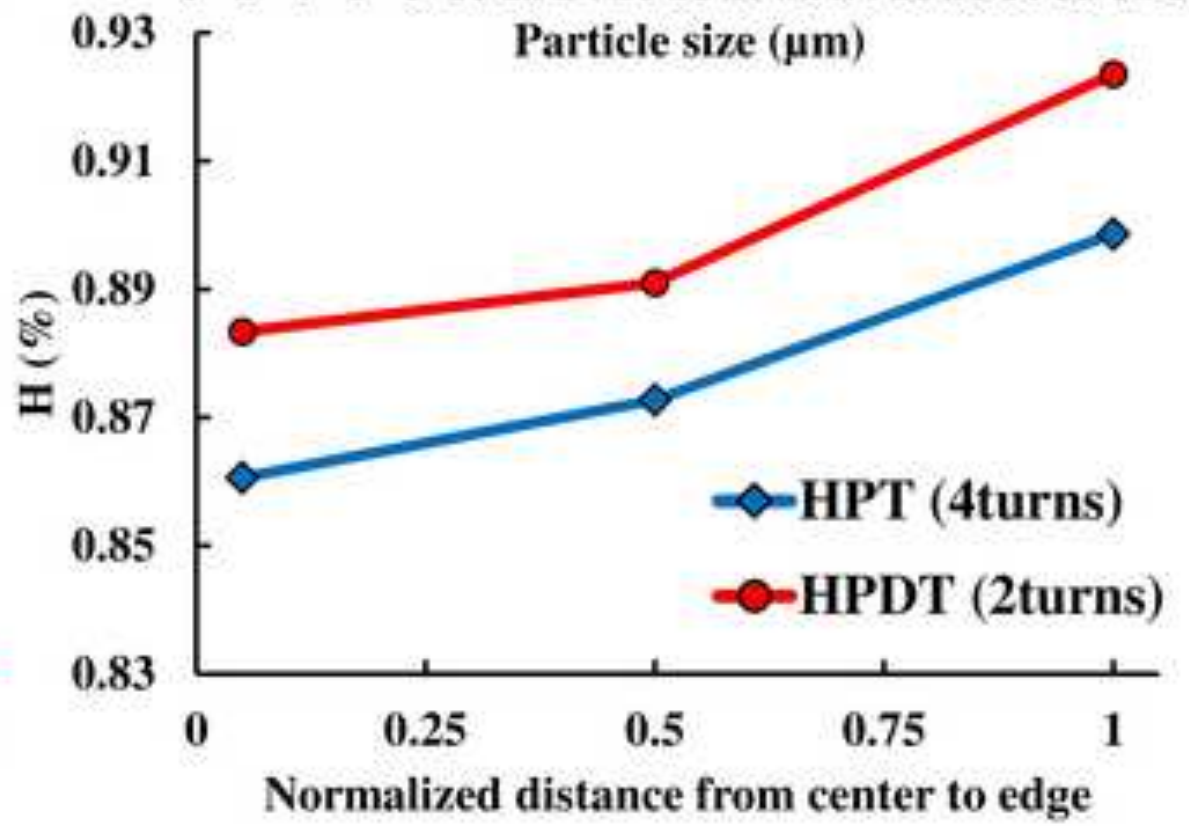




\begin{tabular}{|c|c|c|c|c|c|}
\hline \multirow{2}{*}{$\begin{array}{c}\text { Manufacturing } \\
\text { method }\end{array}$} & \multirow{2}{*}{ \# of turn } & \multicolumn{3}{|c|}{ Location } & \multirow{2}{*}{$\begin{array}{c}P \text {-value } \\
\text { (Radius varies at } \\
\text { constant \# of turns) }\end{array}$} \\
\hline & & Center & Middle & Edge & \\
\hline \multirow{3}{*}{ HPDT } & 2 & 0.8834 & 0.8912 & 0.9235 & 0.026 (Accepted) \\
\hline & 4 & 0.8711 & 0.8533 & 0.8836 & 0.016 (Accepted) \\
\hline & 6 & 0.8771 & 0.8821 & 0.8939 & 0.048 (Accepted) \\
\hline \multicolumn{2}{|c|}{$\begin{array}{c}P \text {-value } \\
\text { (\# of turns varies at } \\
\text { constant radius) }\end{array}$} & $\begin{array}{c}0.693 \\
\text { (Rejected) }\end{array}$ & $\begin{array}{c}0.032 \\
\text { (Accepted) }\end{array}$ & $\begin{array}{c}0.013 \\
\text { (Accepted) }\end{array}$ & \\
\hline \multirow{3}{*}{ HPT } & 2 & 0.8599 & 0.8549 & 0.8768 & 0.572 (Rejected) \\
\hline & 4 & 0.8606 & 0.8727 & 0.8986 & 0.058 (Rejected) \\
\hline & 6 & 0.8570 & 0.8584 & 0.8694 & 0.611 (Rejected) \\
\hline \multicolumn{2}{|c|}{$\begin{array}{c}P \text {-value } \\
\text { (\# of turns varies at } \\
\text { constant radius) }\end{array}$} & $\begin{array}{c}0.938 \\
\text { (Rejected) }\end{array}$ & $\begin{array}{c}0.751 \\
\text { (Rejected) }\end{array}$ & $\begin{array}{c}0.136 \\
\text { (Rejected) }\end{array}$ & \\
\hline
\end{tabular}




\begin{tabular}{|l|c|c|c|}
\hline \multirow{2}{*}{ Manufacturing methods } & \multicolumn{3}{|c|}{ Locations } \\
\cline { 2 - 4 } & Center & Middle & Edge \\
\hline HPDT (2turns) vs. HPT (4turns) & 0.151 & 0.343 & 0.262 \\
\hline
\end{tabular}



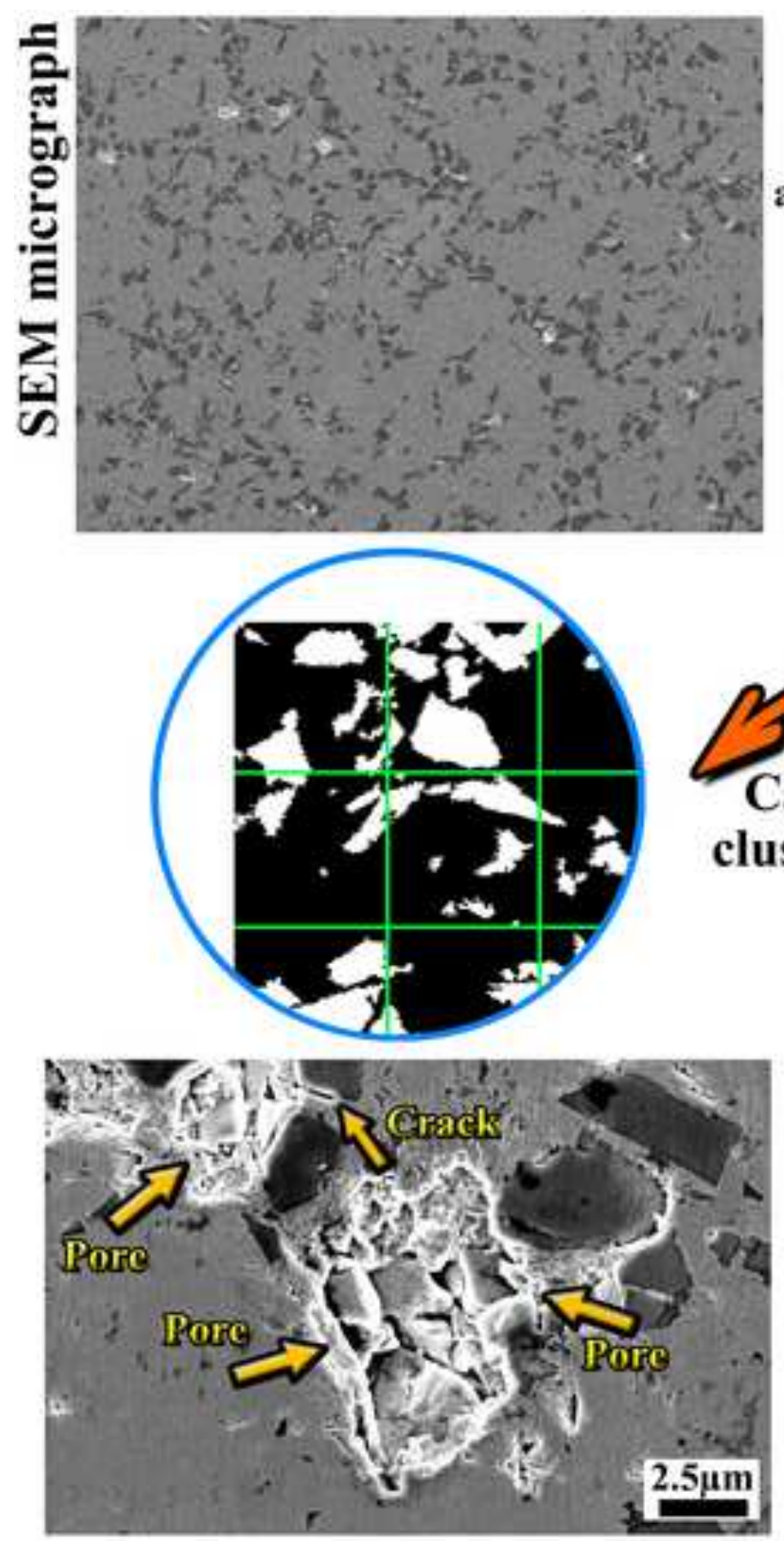

Apply $K$-means algorithm to find the threshold

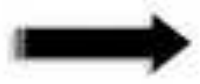

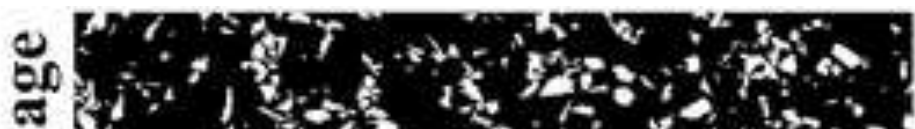

政

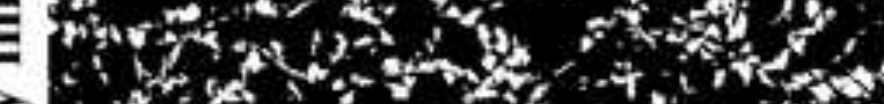

를

.

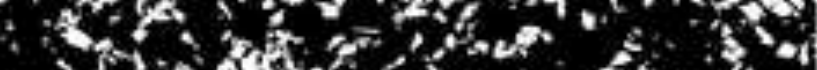

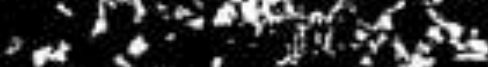

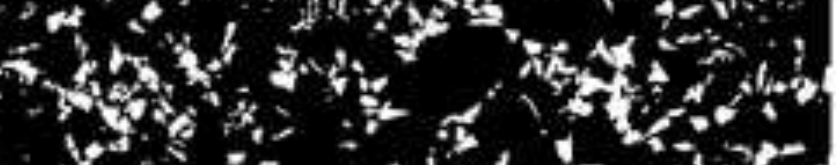

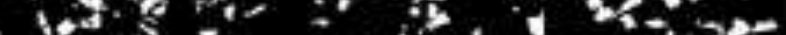

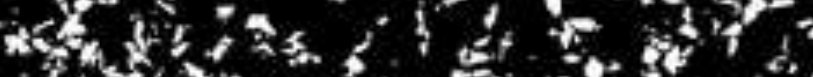
yot

\section{Macro-pixel} analysis

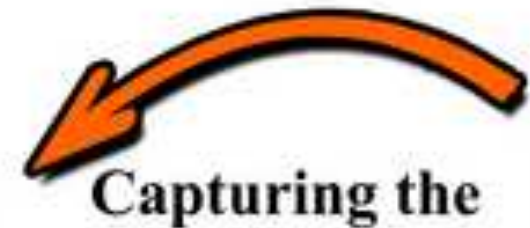
clustered regions
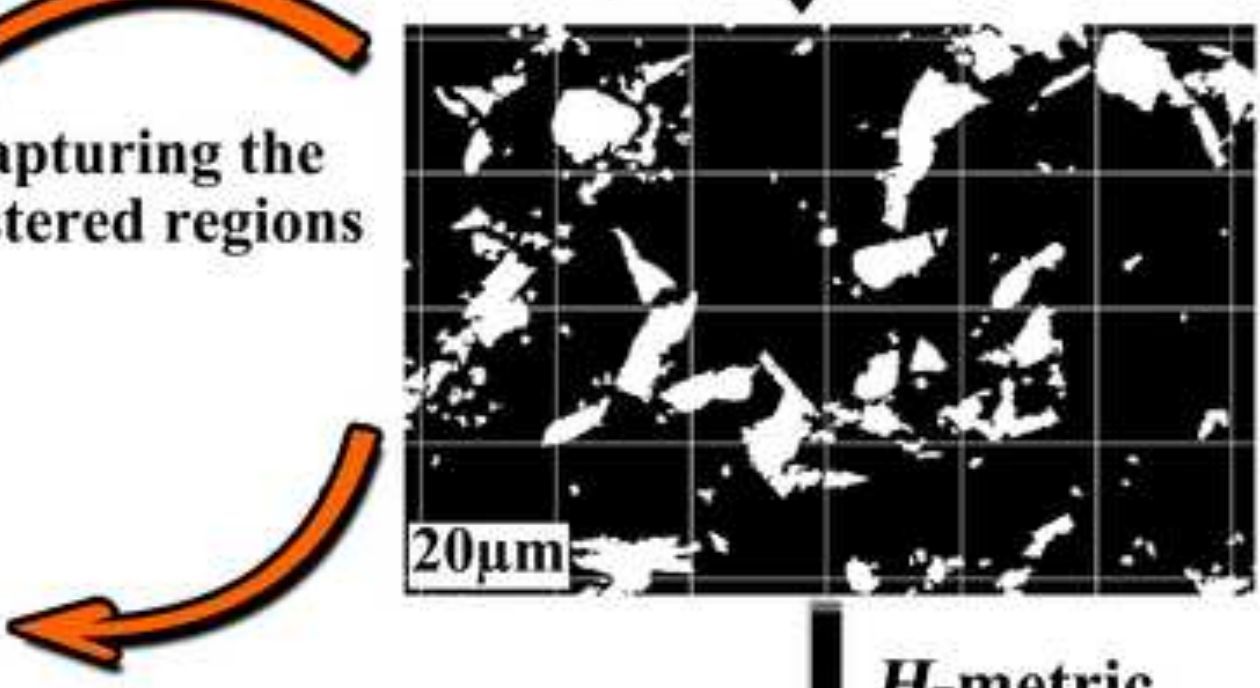

Microstructral defects due to poor distribution
H-metric

$$
H=\frac{\bar{G}_{\text {Sd }}}{\bar{G}_{\text {Sd_random }}}
$$

